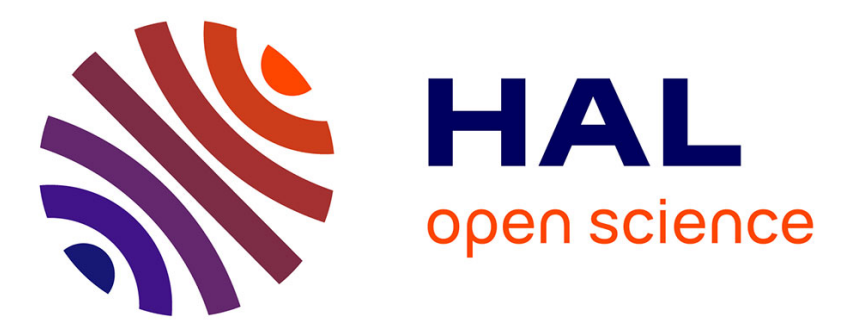

\title{
Dynamic fracture analysis of the linearly uncoupled and coupled physical phenomena by the variable-node multiscale XFEM
}

Shuohui Yin, Ning Zhang, Peng Liu, Jingang Liu, Tiantang Yu, Shuitao Gu, Yu Cong

\section{To cite this version:}

Shuohui Yin, Ning Zhang, Peng Liu, Jingang Liu, Tiantang Yu, et al.. Dynamic fracture analysis of the linearly uncoupled and coupled physical phenomena by the variable-node multiscale XFEM. Engineering Fracture Mechanics, 2021, 254, pp.107941. 10.1016/j.engfracmech.2021.107941 . hal03334432

\section{HAL Id: hal-03334432 \\ https://hal.science/hal-03334432}

Submitted on 3 Sep 2021

HAL is a multi-disciplinary open access archive for the deposit and dissemination of scientific research documents, whether they are published or not. The documents may come from teaching and research institutions in France or abroad, or from public or private research centers.
L'archive ouverte pluridisciplinaire $\mathbf{H A L}$, est destinée au dépôt et à la diffusion de documents scientifiques de niveau recherche, publiés ou non, émanant des établissements d'enseignement et de recherche français ou étrangers, des laboratoires publics ou privés. 


\title{
Dynamic fracture analysis of the linearly uncoupled and coupled physical phenomena by the variable-node multiscale XFEM
}

\author{
Shuohui Yin a,b, Ning Zhang ${ }^{\mathrm{a}}$, Peng Liu ${ }^{\mathrm{c}}$, Jingang Liu ${ }^{\mathrm{a}}$, \\ Tiantang $\mathrm{Yu}^{\mathrm{d}^{*}}$, Shuitao $\mathrm{Gu}^{\mathrm{e} \dagger}$, Yu Cong ${ }^{\mathrm{f}}$ \\ ${ }^{a}$ School of Mechanical Engineering, Xiangtan University, Hunan, 411105, PR China. \\ ${ }^{b}$ Foshan Green Intelligent Manufacturing Research Institute of Xiangtan University, Guangdong 528311, \\ PR China. \\ ${ }^{c}$ State Key Laboratory of Advanced Design and Manufacturing for Vechicle Body, College of Mechanical \\ and Vehicle, Hunan Universiy, Changsha, China,410082. \\ ${ }^{d}$ Department of Engineering Mechanics, Hohai University, Nanjing 210098, PR China. \\ e School of Civil Engineering, Chongqing University, Chongqing 400044, PR China. \\ ${ }^{f}$ Université Paris-Saclay, Univ Evry, LMEE, 91020, Evry, France.
}

\begin{abstract}
This paper aims to develop a variable-node multiscale extended finite element method (V-XFEM) for dynamic fracture analysis of the linearly uncoupled and coupled physical phenomena in a compact formula. The general governing equations for the linearly uncoupled and coupled physical phenomena are presented in a compact form. The local mesh refinement technique for modeling cracks is used to improve the accuracy and efficiency, in which variable-node elements without modifying the system matrix or impose additional boundary conditions are taken to connect/link different scale elements. In addition, the time-dependent equations are solved by the unconditionally stable implicit Newmark time integration method, and the dynamic intensity factors (DIFs) are derived from the domain forms of the interaction integrals. Numerical results of the linearly elastic and piezoelectric problems show that V-XFEM is an efficient numerical approach to simulate the dynamic fracture problems of the linearly uncoupled and coupled physical phenomena.
\end{abstract}

Keywords: Dynamic fracture; Piezoelectric structures; V-XFEM; Variable-node elements; Dynamic stress intensity factor.

\footnotetext{
*Corresponding author Email addresses: tiantangyu@hhu.edu.cn(T. Yu);

†Corresponding author Email addresses: gust@cqu.edu.cn (S.T. Gu);
} 


\begin{tabular}{|c|c|c|c|}
\hline \multicolumn{4}{|c|}{ Nomenclature } \\
\hline$\alpha, \beta$ & physical field subscripts & $K_{i j}$ & magnetoelectric moduli \\
\hline$i, j$ & space subscripts & $\mathbf{w}_{\alpha}^{h}(\boldsymbol{x})$ & enriched field \\
\hline $\mathbf{J}\left(J_{\alpha i}\right)$ & general stress tensor (components) & $N_{\alpha i}(\boldsymbol{x})$ & standard FE shape functions \\
\hline $\mathbf{E}\left(E_{\alpha i}\right)$ & general strain tensor (components) & $\mathbf{w}_{\alpha}^{i}$ & nodal vectors \\
\hline $\mathbf{W}\left(w_{\alpha}\right)$ & $\begin{array}{lll}\begin{array}{l}\text { general displace } \\
\text { (components) }\end{array} & \text { vector } \\
\end{array}$ & $\boldsymbol{a}_{\alpha}^{j}, \boldsymbol{b}_{\alpha I}^{k}$ & $\begin{array}{l}\text { Enriched unknown variables of } \\
\text { nodes }\end{array}$ \\
\hline $\mathbb{L}\left(L_{\alpha i \beta j}\right)$ & general elastic tensor (components) & $N^{s}, N^{c u t}, N^{t i p}$ & $\begin{array}{l}\text { set of all nodes in the whole } \\
\text { discretized domain, associated } \\
\text { with crack-faces and the crack-tips }\end{array}$ \\
\hline $\mathbb{M}\left(M_{\alpha i \beta j}\right)$ & $\begin{array}{l}\text { general compliance tensor } \\
\text { (components) }\end{array}$ & $H(\boldsymbol{x})$ & modified Heaviside step function \\
\hline$\sigma_{i j}$ & Stress components & $F_{I}(\boldsymbol{x})$ & $\begin{array}{lll}\text { crack-tip branch enrichment } \\
\text { functions }\end{array}$ \\
\hline$\varepsilon_{i j}$ & Strain components & $\Delta t$ & time increment \\
\hline$d_{i}$ & electric displacement components & $\xi, \eta$ & $\begin{array}{l}\text { local coordinates in the } \\
\text { isoparametric element }\end{array}$ \\
\hline$h_{i}$ & electric field vector components & $K_{I}, K_{I I}$ & elastic stress intensity factor \\
\hline$m_{i}$ & magnetic flux components & $K_{I V}$ & $\begin{array}{l}\text { electrical displacement intensity } \\
\text { factor }\end{array}$ \\
\hline$a_{i}$ & magnetic field vector components & $K_{I}^{d y n}$ & dynamic stress intensity factor \\
\hline$C_{i j k l}$ & elastic moduli & $\bar{K}_{I}$ & normalized stress intensity factors \\
\hline$D_{i j}$ & dielectric moduli & $\mathrm{a}$ & Crack length \\
\hline$H_{i j}$ & magnetic permeability moduli & $\varphi$ & electric potential \\
\hline$\prod_{k i j}$ & piezoelectric moduli & $\psi$ & magnetic potential \\
\hline$\Lambda_{k i j}$ & piezomagnetic moduli & & \\
\hline
\end{tabular}




\section{Introduction}

An accurate description of the singular fields near the crack-tip is often of great importance in modeling of fracture mechanics problems. However, in contrast to the static loading conditions, numerical simulation of the dynamic fracture problems remains a challenge in many practical engineering applications. Song et al. [1] investigated three finite element methods for dynamic crack propagation, it shows that XFEM and interelement method show similar crack speeds and crack paths, and element deletion method is unable to predict crack branching. The extended finite element method (XFEM) $[2,3]$ is a very powerful numerical tool for modeling arbitrary cracks without remeshing. Recently, it has received much attention in a wide range of engineering problems including the dynamic fracture mechanics problems, e.g., see [4-6]. A detailed overview on the application of extended finite element method in modeling of arbitrary discontinuities can be found in Refs. [7,8].

In fracture mechanics problems, a fine mesh is often required in order to accurately describe the singular fields near the crack-tip and model crack growth path. However, the region containing cracks is usually only a small portion of the whole structure, it is unreasonable to adopt a small-scale mesh in the whole structure due to the computational efficiency. Therefore, numerous multiscale methods which adopt a locally refined mesh have been proposed. In multiscale methods, two-scale meshes are nonconforming, how to link or connect meshes at different level and scales is the main challenge. Guidault et al. $[9,10]$ decomposed the structure into two substructures with distinct scales and linked the two distinct scale meshes by transmitting interactions at multiscale interfaces. Based on a two-scale decomposition, Loehnert and Belytschko [11] proposed a multiscale projection method for macro/microcrack simulations in which macrocracks and microcracks are taken into account by the XFEM. Zhou and Yang [12] and Holl et al. [13,14] adopted the multiscale projection method to simulate propagation and coalescence of multiple cracks. Murotani et al. [15] proposed an adaptive finite element using a hierarchical mesh and applied it to crack propagation analysis. Hettich et al. [16] proposed a variational multiscale method to model failure, in which the Lagrange multipliers was adopted to connect the large and small scales meshes. Dhia and Rateau [17] used the Arlequin method to link fine scale model and a large scale model for simulating crack propagation through the XFEM. For 3D crack propagation in large structures, Rannou et al. [18] developed a local multigrid approach to coupe the grids at different 
scales within the XFEM framework. Gibert et al. [19] presented a multi scale approach coupling the $\mathrm{X}-\mathrm{FEM}$ method with automatic adaptive mesh refinement for 2D and 3D fatigue crack propagation. But, a projection of the mechanical state from one mesh to another during the propagation is necessary. Fries et al. [20] proposed a XFEM with locally refined mesh through two kinds of hanging nodes which have or have no degrees of freedom. Budarapu et al. [21] developed an adaptive multiscale method in which the different scales are connected by ghost atoms. Patil et al. [22] constructed a multiscale XFEM for modeling heterogeneous materials using the multiscale basis functions as a bridge between two scales. Teng et al. [23] integrated the virtual node polygonal elements with XFEM for modelling dynamic crack growth. Kumar et al. [24] developed a homogenized multigrid XFEM for the crack growth simulations in ductile materials, in which the domain contains the microstructural defects is simulated by fine mesh while other domains are simulated by coarse mesh with homogenized properties. Among those methods, extra efforts such as modification on the system matrix or imposing additional boundary conditions are unavoidable.

To overcome this numerical problem, the variable-node elements [25] with an arbitrary number of nodes on each of their edges were developed based on the generic point interpolation, which can provide a flexibility to connect different scale meshes without modify the system matrix or impose additional boundary conditions. Recently, the variable-node XFEM (V-XFEM) has been developed for modeling cracks, voids, and inclusions in both 2D and 3D elastic mediums [26-28] by introducing the variable-node elements into the traditional XFEM codes. Due to its advantages of having no additional constraints, the variable-node elements can be conveniently embedded into the traditional XFEM codes. The V-XFEM has been proved to be a general and efficient approach in the framework of multiscale methods, and achieves a faster convergence rate in energy norm error than the standard XFEM [28]. Ding et al. [29, 30] present a Matlab object-oriented implementation of an efficient V-XFEM for the problems of multiple crack growth simulation and strong and weak discontinuities. Han et al. [31] proposed a triangular extended stochastic finite element method (T-XSFEM) for simulation of random void problems with the aid of variable-node elements to couple/link different mesh-scales. More recently, the V-XFEM are extended for detection of multiple complicated flaw clusters in large structures [32]. Even much efforts in the fracture analyses, to the best of the authors' knowledge, the attention has not been paid to the dynamic fracture problems. 
Piezoelectric materials have been widely used as actuators, transducers or sensors because of the inherent coupling effect between mechanical and electric fields. Piezoelectric materials possess many advantages but suffer the brittleness and low fracture toughness. As the growing demand for wide range of applications of piezoelectric materials, the dynamic fracture problem has been a serious concern nowadays for the design engineers of piezoelectric structures. Li and Mataga [33] studied transient response of a semi-infinite, anti-plane crack propagating in a hexagonal piezoelectric medium by transform methods together with the Wiener-Hopf and Cagniardde Hoop techniques. However, deriving analytical solutions for time dependent problems of cracks under dynamic impact loading condition are very restricted and numerical methods are required for practical application of piezoelectric structures. Therefore, Enderlein et al. [34] applied finite element method (FEM) to model dynamic fracture problems in piezoelectric materials. Studies on fracture problems in piezoelectric materials under dynamic loading have also modeled by other numerical approaches including the BEM [35,36], meshless methods[37], scaled boundary finite element method (SBFEM) [38] and the extended finite element method (XFEM)[4-6, 39]. Bechet et al. [40] devised an enrichment scheme with six crack tip functions based on the asymptotic expansion around a crack tip in piezoelectric materials.

In the present paper, we extend the applicability of V-XFEM to the dynamic fracture analysis for the linearly uncoupled and coupled physical phenomena in a compact formula by introducing the general governing equations. The time-dependent equations to be solved by the unconditionally stable implicit Newmark time integration method and the dynamic intensity factors (DIFs) are derived from the domain forms of the interaction integrals. The numerical results of the elastic and piezoelectric problems considered as illustrated examples are discussed in order to show the efficiency and accuracy of V-XFEM. Thus, the main features of the present work consists of: (i) extension of V-XFEM to the dynamic fracture simulation, (ii) treatment of problem for all the linear uncoupled phenomena as an uncoupled problem in a unified and compact way.

The rest of the paper is structured as follows. The formulation of general governing equations is given in Section 2. Considering piezoelectric structures as illustrated examples, the V-XFEM is elaborated in detailed for dynamic crack problem including solution of dynamic fracture, variable-node elements and evaluation of generalized dynamic intensity factors in Section 3. Section 4 the numerical illustrations are given for several elastic and piezoelectric problems, which 
show that the obtained solutions agree well with results available in the literature and V-XFEM has more efficient than the standard XFEM. Finally, some conclusions are summarized in Section 5.

\section{General governing equations}

In this section, we present general governing equations for the linearly uncoupled and coupled physical phenomena. Firstly, we introduce two 3D vector fields $\boldsymbol{J}_{\alpha}$ and $\boldsymbol{E}_{\alpha}$ written by

$$
\begin{aligned}
& \operatorname{div} \boldsymbol{J}_{\alpha}=J_{\alpha i, i}=\gamma_{\alpha}(t), \\
& \boldsymbol{E}_{\alpha}=\nabla \omega_{\alpha} \text { or } E_{\alpha i}=\omega_{\alpha, i} .
\end{aligned}
$$

Then, the constitutive relations for all the known linearly uncoupled phenomena like elasticity and the linearly coupled phenomena like piezoelectricity and magneto-electro-elasticity can be written in a unified way (see, e.g. $[41,42])$

$$
\boldsymbol{J}_{\alpha}=\boldsymbol{L}_{\alpha \beta} \boldsymbol{E}_{\beta} \text { or } J_{\alpha i}=L_{\alpha i \beta j} E_{\beta j}
$$

where the Greek letters $\alpha$ and $\beta$ indicate field subscripts assuming the values 1 to $r$, the Latin letters $i$ and $j$ represent space subscripts taking the values 1 and 2 for two dimensional problem or 1 , 2 and 3 for three dimensional problem, and $\omega_{\alpha}$ are the components of an $r$-dimensional potential vector $\mathbf{w}$. It is often rewritten as

$$
\mathbf{J}=\mathbb{L} \mathbf{E}
$$

where $\mathbf{J}$ and $\mathbf{E}$ can be viewed as two vectors of $3 r$ components and $\mathbb{L}$ is a $3 r \times 3 r$ matrix such that

$$
\mathbf{J}=\left[\begin{array}{l}
\boldsymbol{J}_{1} \\
\boldsymbol{J}_{2} \\
\vdots \\
\boldsymbol{J}_{r}
\end{array}\right], \quad \mathbb{L}=\left[\begin{array}{cccc}
\boldsymbol{L}_{11} & \boldsymbol{L}_{12} & \cdots & \boldsymbol{L}_{1 r} \\
\boldsymbol{L}_{21} & \boldsymbol{L}_{22} & \cdots & \boldsymbol{L}_{2 r} \\
\vdots & \vdots & \vdots & \vdots \\
\boldsymbol{L}_{r 1} & \boldsymbol{L}_{r 2} & \cdots & \boldsymbol{L}_{r r}
\end{array}\right], \quad \mathbf{E}=\left[\begin{array}{l}
\boldsymbol{E}_{1} \\
\boldsymbol{E}_{2} \\
\vdots \\
\boldsymbol{E}_{r}
\end{array}\right]
$$

Here, an element $\boldsymbol{L}_{\alpha \beta}$ of $\mathbb{L}$ is a $3 \times 3$ matrix. In addition, the matrix $\mathbb{L}$ is invertible, so that the dual expression of (4) is

$$
\mathbf{E}=\mathbb{M} \mathbf{J}
$$

with $\mathbb{M}=\mathbb{L}^{-1}$. It should be noted that these above formulations allow us to systematically treat the problem for all the linear uncoupled phenomena as an uncoupled problem. 
Let us consider the magneto-electro-elasticity as an example for the foregoing formulation Eq. (4). The constitutive law of this problem is usually defined by (see, e.g. [43])

$$
\left\{\begin{array}{l}
\sigma_{i j}=C_{i j k l} \varepsilon_{k l}-\prod_{k i j} h_{k}-\Lambda_{k i j} a_{k} \\
d_{i}=\prod_{i k l} \varepsilon_{k l}+D_{i j} h_{j}+K_{i j} a_{j} \\
m_{i}=\Lambda_{i k l} \varepsilon_{k l}+K_{i j} h_{j}+H_{i j} a_{j}
\end{array}\right.
$$

where $\sigma_{i j}$ and $\varepsilon_{i j}$ are the components of the stress and strain tensors; $d_{i}$ and $h_{i}$ are the components of the electric displacement and electric field vectors; $m_{i}$ and $a_{i}$ are the components of the magnetic flux and magnetic field vectors. Here, $C_{i j k l}, D_{i j}$ and $H_{i j}$ are the elastic, dielectric and magnetic permeability moduli; $\Pi_{k i j}, \Lambda_{k i j}$ and $K_{i j}$ are the piezoelectric, piezomagnetic and magnetoelectric moduli. These material moduli have the following symmetry properties:

$$
\begin{gathered}
C_{i j k l}=C_{i j l k}=C_{k l i j}, D_{i j}=D_{j i}, H_{i j}=H_{j i}, \\
K_{i j}=K_{j i}, \prod_{k i j}=\prod_{k j i}, \Lambda_{k i j}=\Lambda_{k j i}
\end{gathered}
$$

The strain, electric field, and magnetic fields can be derivable from a displacement field $\boldsymbol{u}$, electric potential $\varphi$, and magnetic potential $\psi$, respectively, as follows:

$$
\varepsilon_{i j}=\frac{1}{2}\left(u_{i, j}+u_{j, i}\right), h_{i}=-\varphi_{, i}, a_{i}=-\psi_{, i}
$$

In addition, for simplifying our analysis, inertia effects are considered and other unsteady effects are ignored. Thus, the stress tensor $\boldsymbol{\sigma}$, the electric displacement vector $\boldsymbol{d}$ and the magnetic flux vector b satisfy:

$$
\sigma_{i j, j}=\rho \frac{\partial^{2} u_{i}}{\partial t^{2}}, d_{i, i}=0, b_{i, i}=0
$$

The magneto-electro-thermo-elastic constitutive law (7) can be recast into the compact form Eq. (5) as a coupled field phenomena with five coupled fields $(r=5)$. More precisely, the following definitions are adopted:

$$
\begin{gathered}
J_{1 i}=\sigma_{1 i}, \quad J_{2 i}=\sigma_{2 i}, \quad J_{3 i}=\sigma_{3 i}, J_{4 i}=d_{i}, J_{5 i}=b_{i} \\
E_{\alpha i}=\omega_{\alpha, i}, \boldsymbol{w}=\left(\begin{array}{lllll}
u_{1} & u_{2} & u_{3} & \varphi & \psi
\end{array}\right)^{T}
\end{gathered}
$$




$$
\mathbb{L}=\left[L_{\alpha \beta}\right]=\left[\begin{array}{ccccc}
C_{i 1 j 1} & C_{i 1 j 2} & C_{i 1 j 3} & \prod_{j i 1} & \Lambda_{j i 1} \\
C_{i 2 j 1} & C_{i 2 j 2} & C_{i 2 j 3} & \prod_{j i 2} & \Lambda_{j i 2} \\
C_{i 3 j 1} & C_{i 3 j 2} & C_{i 3 j 3} & \prod_{j i 3} & \Lambda_{j i 3} \\
\prod_{i 1 j} & \prod_{i 2 j} & \prod_{i 3 j} & -D_{i j} & -K_{i j} \\
\Lambda_{i 1 j} & \Lambda_{i 2 j} & \Lambda_{i 3 j} & -K_{j i} & -H_{i j}
\end{array}\right]
$$

with

$$
\gamma(t)=\left(\rho \frac{\partial^{2} u_{i}}{\partial t^{2}}, 0,0\right)
$$

The general prescribed conditions on the boundary $S$ consisting of $S_{J}$ and $S_{w}$ can be expressed by

$$
J_{\alpha}=J_{\alpha i} n_{i}=\bar{J}_{\alpha}\left(\text { on } S_{J}\right) \text { and } \boldsymbol{w}_{\alpha}=\overline{\boldsymbol{w}}_{\alpha}\left(\text { on } S_{w}\right)
$$

where $\boldsymbol{n}$ is the unit outward normal on $S$. And, the crack-faces in this study are assumed to be general traction-free, i.e.

$$
J_{\alpha i} v_{i}=0
$$

Here, $v$ is the unit vector normal to the crack-faces $S_{c}$.

The weak form of the general governing equations is gained from Hamilton's variational principle:

$$
\delta \int_{\mathbf{t}_{0}}^{\mathbf{t}_{1}} \int_{\mathbf{V}}\left[\frac{1}{2} \rho(\dot{\mathbf{u}})^{2}-\frac{1}{2}(\mathbf{E} \mathbb{L} \mathbf{E})\right] d V d t+\int_{\mathbf{t}_{0}}^{\mathbf{t}_{1}} J_{\alpha} \delta \mathbf{w}_{\alpha} d t=0
$$

After doing some basic calculus, the above equation finally yields the weak form as follows:

$$
-\int_{\mathbf{t}_{0}}^{\mathrm{t}_{1}} \int_{\mathbf{V}}\left[\rho \ddot{\mathbf{i}} \delta \mathbf{u}^{2}+(\mathbf{E} \mathbb{L} \delta \mathbf{E})\right] d V d t+\int_{\mathbf{t}_{0}}^{\mathrm{t}_{1}} J_{\alpha} \delta \mathbf{w}_{\alpha} d t=0
$$

Here, the superscript $\bullet$ means the derivative with respect to time.

It should be noted that the general weak form for the linearly uncoupled and coupled physical phenomena is expressed in a compact form. In the following, the elastic and piezoelectric problems will be considered as linearly uncoupled and coupled physical examples, respectively, for the dynamic fracture analysis.

\section{V-XFEM for dynamic crack problem in linearly elastic and piezoelectric structures}

Now, we proceed to elaborate a V-XFEM to simulate the dynamic fracture in the problem formulated in the previous section. Thus, we particularize the general governing equations to the 
linearly elasticity and piezoelectricity. It should be noted that the linearly piezoelectric problems can reduce to linearly elastic problems by ignoring the coupled piezoelectric tensor and suppressing the electric field and the electric displacements. In order not to be redundant, the corresponding formulations associated to piezoelectricity are only described in the following.

\subsection{Enriched field approximation}

In the X-FEM [2, 3], the standard finite element approximation is enriched by additional functions based on the partition of unity to model the field discontinuities. For piezoelectric materials, Bechet et al. [40] devised an enrichment scheme with six crack tip functions. A comparison of the normalized dynamic intensity factors with the six crack tip enrichments and the standard four crack tip enrichments is presented in this paper. It shows that the results are matched very well and consistent with the conclusion of Bechet et al [40] that the four-fold enrichment is almost as efficient, concerning accuracy both in energy and in the SIFs. However, the four-fold enrichment is simpler to implement and involves less computational overhead, because it adds only four degrees of freedom (dofs) per regular dof, instead of six. Therefore, the four-fold enrichment is adopted in this paper. With general governing equations, the enriched field $\mathbf{w}_{\alpha}^{h}(\boldsymbol{x})$ can be expressed in the form:

$$
\mathbf{w}_{\alpha}^{h}(\boldsymbol{x})=\sum_{i \in N^{s}} N_{\alpha i}(\boldsymbol{x}) \mathbf{w}_{\alpha}^{i}+\sum_{j \in N^{\text {cut }}} N_{\alpha j}(\boldsymbol{x}) H(\boldsymbol{x}) \boldsymbol{a}_{\alpha}^{j}+\sum_{k \in N^{t i p}} N_{\alpha k}(\boldsymbol{x}) \sum_{I=1}^{4} F_{I}(\boldsymbol{x}) \boldsymbol{b}_{\alpha I}^{k} .
$$

In the above equation, $N_{\alpha i}(\boldsymbol{x})$ are the standard finite element shape functions; $\mathbf{w}_{\alpha}^{i}$ are the nodal vectors defined in standard finite elements; $\boldsymbol{a}_{\alpha}^{j}$ and $\boldsymbol{b}_{\alpha I}^{k}$ are the enriched unknown variables of nodes; $N^{s}, N^{\text {cut }}$ and $N^{\text {tip }}$ are the set of all nodes in the whole discretized domain, the set of enriched nodes associated with crack-faces and the set of the enriched nodes associated with the crack-tips, respectively; $H(\boldsymbol{x})$ is a modified Heaviside step function (jump function), which takes the value of +1 above the crack while -1 below the crack; $F_{I}(\boldsymbol{x})$ is the crack-tip branch enrichment functions.

Obviously, in the piezoelectric problem, four vector fields $(r=4)$ involves with 
$\mathbf{w}_{\alpha}=\left(\begin{array}{llll}u_{1} & u_{2} & u_{3} & \varphi\end{array}\right)^{T}$. In this case, the enriched displacements and electrical potential approximation for the crack problem involve. That means Eq. (19) can be rewritten by

$$
\begin{aligned}
\boldsymbol{u}^{h}(\boldsymbol{x}) & =\sum_{i \in N^{s}} N_{i}(\boldsymbol{x}) \boldsymbol{u}_{i}+\sum_{j \in N^{\text {cut }}} N_{j}(\boldsymbol{x}) H(\boldsymbol{x}) \boldsymbol{a}_{j}+\sum_{k \in N^{\text {tip }}} N_{k}(\boldsymbol{x}) \sum_{I=1}^{4} F_{I}(\boldsymbol{x}) \boldsymbol{b}_{k I}, \\
\varphi^{h}(\boldsymbol{x}) & =\sum_{i \in N^{s}} N_{i}(\boldsymbol{x}) u_{i}^{*}+\sum_{j \in N^{\text {cut }}} N_{j}(\boldsymbol{x}) H(\boldsymbol{x}) a_{j}^{*}+\sum_{k \in N^{\text {tip }}} N_{k}(\boldsymbol{x}) \sum_{I=1}^{4} F_{I}(\boldsymbol{x}) \boldsymbol{b}_{k I}^{*} .
\end{aligned}
$$

In addition, with reference to these works [4, 44], the corresponding crack-tip branch enrichment functions are taken as

$$
\left\{F_{I}(\boldsymbol{x})\right\}_{I=1}^{4}=\left\{\sqrt{r} \sin \frac{\theta}{2} \quad \sqrt{r} \cos \frac{\theta}{2} \quad \sqrt{r} \sin \frac{\theta}{2} \sin \theta \quad \sqrt{r} \cos \frac{\theta}{2} \sin \theta\right\}
$$

where $r$ and $\theta$ are the usual crack-tip polar co-ordinates.

We need to emphasize that Eq. (19) can similarly be reduced to some prescribed formulation associated to the linearly physical phenomena under consideration.

\subsection{Numerical discretization}

In order to discretize the linearly piezoelectric problem, in the case of neglecting of damping, substituting the enriched approximated functions in Eq. (20) into the weak-form of the equilibrium equations as illustrated in [45] and after some appropriate manipulations, a system of discretized piezoelectric XFEM equations can be obtained as follows:

$$
\tilde{M} \ddot{\zeta}+K \zeta=F
$$

where $\zeta$ denotes the nodal unknowns, and $\tilde{\boldsymbol{M}}, \boldsymbol{K}$ and $\boldsymbol{F}$ are the generalized global mass matrix, global stiffness matrix and external force vector, respectively, with $\tilde{\boldsymbol{M}}=\left[\begin{array}{cc}\boldsymbol{M} & 0 \\ 0 & 0\end{array}\right], \quad \boldsymbol{\zeta}=\left\{\begin{array}{l}\boldsymbol{\delta} \\ \chi\end{array}\right\}$, $\boldsymbol{F}=\left\{\begin{array}{l}\boldsymbol{F}^{\text {mech }} \\ \boldsymbol{F}^{\text {elec }}\end{array}\right\}$, and $\boldsymbol{\delta}=\left[\begin{array}{lll}\boldsymbol{u} & \boldsymbol{a} & \boldsymbol{b}\end{array}\right]^{T}, \boldsymbol{\chi}=\left[\begin{array}{lll}\boldsymbol{u}^{*} & \boldsymbol{a}^{*} & \boldsymbol{b}^{*}\end{array}\right]^{T}$. Analogously, substituting the enriched approximated functions in Eq. (19) into the weak-form of the general equilibrium equations, a system of discretized equations for all the linear uncoupled phenomena as an uncoupled problem can be obtained by introducing the corresponding number of physical fields.

In the linearly piezoelectric problem, the element contribution to mass and stiffness matrices 
(superscript $e$ ) are as follows:

$$
\boldsymbol{m}_{i j}^{e}=\left[\begin{array}{ccc}
\boldsymbol{m}_{i j}^{u u} & \boldsymbol{m}_{i j}^{u a} & \boldsymbol{m}_{i j}^{u b} \\
\boldsymbol{m}_{i j}^{a u} & \boldsymbol{m}_{i j}^{a a} & \boldsymbol{m}_{i j}^{a b} \\
\boldsymbol{m}_{i j}^{b u} & \boldsymbol{m}_{i j}^{b a} & \boldsymbol{m}_{i j}^{b b}
\end{array}\right] ; \quad \boldsymbol{k}_{i j}^{e}=\left[\begin{array}{ccc}
\boldsymbol{k}_{i j}^{u u} & \boldsymbol{k}_{i j}^{u a} & \boldsymbol{k}_{i j}^{u b} \\
\boldsymbol{k}_{i j}^{a u} & \boldsymbol{k}_{i j}^{a a} & \boldsymbol{k}_{i j}^{a b} \\
\boldsymbol{k}_{i j}^{b u} & \boldsymbol{k}_{i j}^{b a} & \boldsymbol{k}_{i j}^{b b}
\end{array}\right]
$$

where

$$
\begin{gathered}
\boldsymbol{m}_{i j}^{u u}=\int_{\Omega} \rho\left(\boldsymbol{N}_{i}\right)^{T} \boldsymbol{N}_{j} d \Omega \\
\boldsymbol{m}_{i j}^{a a}=\int_{\Omega} \rho H^{2}\left(\boldsymbol{N}_{i}\right)^{T} \boldsymbol{N}_{j} d \Omega \\
\boldsymbol{m}_{i j}^{b b}=\int_{\Omega} \rho\left(F_{I}\right)^{2}\left(\boldsymbol{N}_{i}\right)^{T} \boldsymbol{N}_{j} d \Omega ;(I=1,2,3,4) \\
\boldsymbol{m}_{i j}^{u a}=\int_{\Omega} \rho H\left(\boldsymbol{N}_{i}\right)^{T} \boldsymbol{N}_{j} d \Omega \\
\boldsymbol{m}_{i j}^{u b}=\int_{\Omega} \rho F_{I}\left(\boldsymbol{N}_{i}\right)^{T} \boldsymbol{N}_{j} d \Omega ;(I=1,2,3,4) \\
\boldsymbol{m}_{i j}^{a b}=\int_{\Omega} \rho H F_{I}\left(\boldsymbol{N}_{i}\right)^{T} \boldsymbol{N}_{j} d \Omega ;(I=1,2,3,4)
\end{gathered}
$$

and

$$
\boldsymbol{k}_{i j}^{r s}=\int_{\Omega_{e}}\left(\boldsymbol{B}_{i}^{r}\right)^{T} \boldsymbol{D} \boldsymbol{B}_{j}^{s} d \Omega ;(r, s=u, a, b)
$$

with

$$
\begin{gathered}
\boldsymbol{B}_{i}^{u}=\left[\begin{array}{ccc}
N_{i, x} & 0 & 0 \\
0 & N_{i, y} & 0 \\
N_{i, y} & N_{i, x} & 0 \\
0 & 0 & N_{i, x} \\
0 & 0 & N_{i, y}
\end{array}\right] \\
\boldsymbol{B}_{i}^{a}=\left[\begin{array}{ccc}
H N_{i, x} & 0 & 0 \\
0 & H N_{i, y} & 0 \\
H N_{i, y} & H N_{i, x} & 0 \\
0 & 0 & H N_{i, x} \\
0 & 0 & H N_{i, y}
\end{array}\right] \\
\boldsymbol{B}_{i}^{b}=\left[\begin{array}{llll}
\boldsymbol{B}_{i}^{b 1} & \boldsymbol{B}_{i}^{b 2} & \boldsymbol{B}_{i}^{b 3} & \boldsymbol{B}_{i}^{b 4}
\end{array}\right]
\end{gathered}
$$




$$
\boldsymbol{B}_{i}^{b I}=\left[\begin{array}{ccc}
\left(N_{i} F_{I}\right)_{, x} & 0 & 0 \\
0 & \left(N_{i} F_{I}\right)_{, y} & 0 \\
\left(N_{i} F_{I}\right)_{, y} & \left(N_{i} F_{I}\right)_{, x} & 0 \\
0 & 0 & \left(N_{i} F_{I}\right)_{, x} \\
0 & 0 & \left(N_{i} F_{I}\right)_{, y}
\end{array}\right]
$$

where $\boldsymbol{D}$ is generalized elastic coefficient matrix in eq. (13).

Additionally, the element contribution to external force vector $\boldsymbol{F}^{\text {mech }}$ is given by

$$
\boldsymbol{f}_{i}^{\text {mech }}=\left[\begin{array}{lll}
\boldsymbol{f}_{i}^{u} & \boldsymbol{f}_{i}^{a} & \boldsymbol{f}_{i}^{b}
\end{array}\right]^{T}
$$

in which

$$
\begin{gathered}
\boldsymbol{f}_{i}^{u}=\int_{\partial \Omega_{e}} \boldsymbol{N}_{i} \boldsymbol{t} d \Gamma \\
\boldsymbol{f}_{i}^{a}=\int_{\partial \Omega_{e}} \boldsymbol{N}_{i} H \boldsymbol{t} d \Gamma, \\
\boldsymbol{f}_{i}^{b I}=\int_{\partial \Omega_{e}} \boldsymbol{N}_{i} F^{I} \boldsymbol{t} d \Gamma ;(I=1,2,3,4 .)
\end{gathered}
$$

The external nodal charge vector $\boldsymbol{F}^{\text {elec }}$ can be computed in a similar manner as above.

It is noted that the Gauss quadrature scheme is employed for non-enrich elements and $4 \times 4$ Gauss quadrature is employed for blending elements. To obtain an accurate integration for crack tip elements and elements cut by crack the triangular sub-domain technique [46] is used in the same way as that of the XFEM.

In this study, the unconditionally stable implicit Newmark method is adopted to solve the discrete dynamic equilibrium equations of Eq. (22). At time step $n$, the discretized equations Eq. (22) is thus rewritten as

$$
\left(\tilde{\boldsymbol{M}}+\beta \Delta t^{2} \boldsymbol{K}\right) \ddot{\boldsymbol{\zeta}}_{n}=\boldsymbol{F}-\boldsymbol{K}\left[\boldsymbol{\zeta}_{n-1}+\Delta t \dot{\boldsymbol{\zeta}}_{n-1}+(1-2 \beta) \frac{\Delta t^{2}}{2} \ddot{\boldsymbol{\zeta}}_{n-1}\right]
$$

in which the velocity and displacement can be evaluated by

$$
\begin{gathered}
\dot{\boldsymbol{\zeta}}_{n}=\dot{\boldsymbol{\zeta}}_{n-1}+\left[(1-\alpha) \ddot{\boldsymbol{\zeta}}_{n-1}+\alpha \ddot{\boldsymbol{\zeta}}_{n}\right] \Delta t \\
\boldsymbol{\zeta}_{n}=\boldsymbol{\zeta}_{n-1}+\Delta t \dot{\boldsymbol{\zeta}}_{n-1}+\left[\left(\frac{1}{2}-\beta\right) \ddot{\boldsymbol{\zeta}}_{n-1}+\beta \ddot{\boldsymbol{\zeta}}_{n}\right] \Delta t^{2}
\end{gathered}
$$

where $\Delta t$ denotes the time increment at the present time-step and the unconditionally stable parameter are $\alpha=0.5$ and $\beta=0.25$. 


\subsection{Variable-node elements}

In order to improve computing efficiency and accuracy, multiscale mesh, i.e., mesh locally refined in the vicinity of cracks, is used and the variable-node elements [23, 26] are adopted to as linking elements (or transition elements) between large scale elements and small scale elements, which is schematically sketched in Fig. 1.

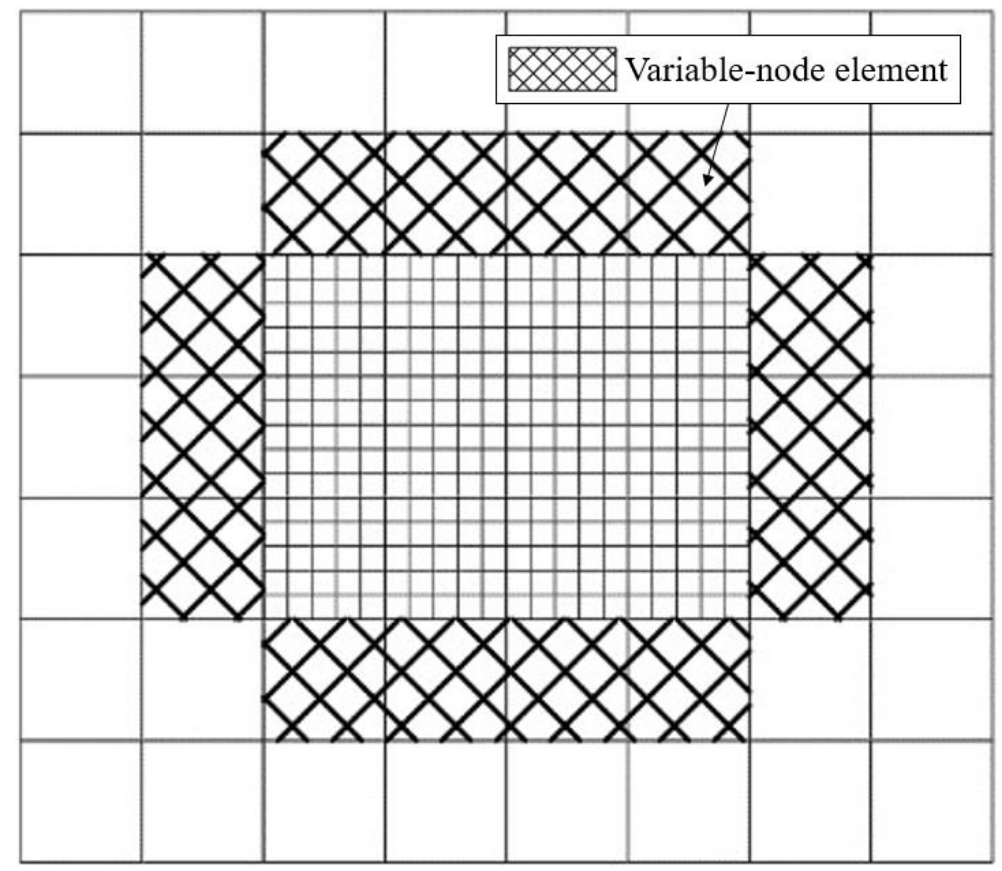

Fig. 1. Illustration of multiscale mesh.

The advantage of variable-node elements is any number of nodes can be attached to the edges of the quadrilateral isoparametric element which is shown in Fig. 2 and the interpolation property between any two adjacent nodes remains linear as the number of nodes in an element increases. Therefore, variable-node elements are suitable for linking between small scale mesh and large scale mesh. 


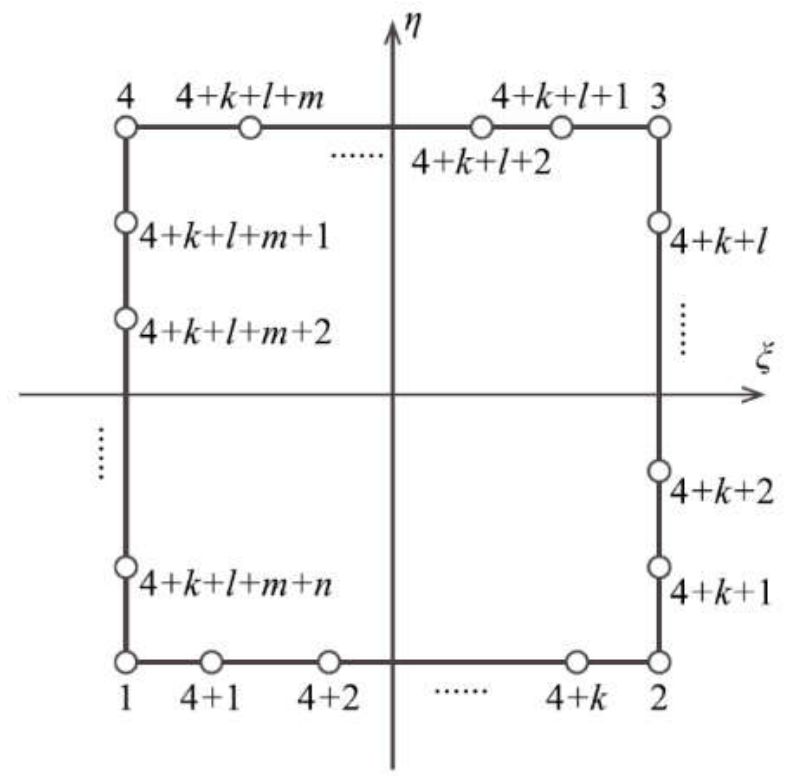

Fig. 2. Illustration of an $(4+k+l+m+n)$-node element.

The approximation displacement $\boldsymbol{u}^{h}(\xi)$ of the variable-node element is given as:

$$
\boldsymbol{u}^{h}(\xi)=\sum_{i=1}^{N_{p}} N_{i}(\xi) \boldsymbol{u}_{i}=\boldsymbol{a}^{T} \boldsymbol{p}(\xi)
$$

where $N_{p}$ is the number of nodes; $\boldsymbol{N}_{i}=\left[\begin{array}{cc}N_{i} & 0 \\ 0 & N_{i}\end{array}\right]$ is the shape function matrix with $N_{i}$ is the shape function of the $i$-th node; $\boldsymbol{u}_{i}=\left[\begin{array}{ll}u_{i} & v_{i}\end{array}\right]^{T}$ is the $i$-th nodal displacement vector; $\boldsymbol{a}^{T}$ is the $2 \times N_{p}$ unknown coefficients matrix and $\boldsymbol{p}(\boldsymbol{\xi})$ is a column vector of the polynomial basis given by [23] 


$$
\boldsymbol{p}(\boldsymbol{\xi})=\left[\begin{array}{c}
1 \\
\xi \\
\eta \\
\xi \eta \\
\left|\xi-\xi_{5}\right|\left(\eta+s i g n \eta_{5}\right) \\
\ldots \\
\left|\xi-\xi_{4+k}\right|\left(\eta+s i g n \eta_{4+k}\right) \\
\left|\eta-\eta_{4+k+1}\right|\left(\xi+s i g n \xi_{4+k+1}\right) \\
\ldots \\
\left|\eta-\eta_{4+k+l}\right|\left(\xi+s i g n \xi_{4+k+l}\right) \\
\left|\xi-\xi_{4+k+l+1}\right|\left(\eta+s i g n \eta_{4+k+l+1}\right) \\
\cdots \\
\left|\xi-\xi_{4+k+l+m}\right|\left(\eta+s i g n \eta_{4+k+l+m}\right) \\
\left|\eta-\eta_{4+k+l+m+1}\right|\left(\xi+s i g n \xi_{4+k+l+m+1}\right) \\
\cdots \\
\left|\eta-\eta_{4+k+l+m+n}\right|\left(\xi+s i g n \xi_{4+k+l+m+n}\right)
\end{array}\right]
$$

in which $\xi$ and $\eta$ are the local coordinates in the isoparametric element.

Using the point interpolation, the approximated displacements is rewritten as

$$
\boldsymbol{u}^{h}(\xi)=\boldsymbol{a}^{T} \boldsymbol{p}(\boldsymbol{\xi})=\boldsymbol{U}^{T} \boldsymbol{q}^{-1} \boldsymbol{p}(\boldsymbol{\xi})
$$

where $\boldsymbol{q}=\left[\boldsymbol{p}\left(\boldsymbol{\xi}_{1}\right), \ldots, \boldsymbol{p}\left(\boldsymbol{\xi}_{4+k+l+m+n}\right)\right]$ and $\boldsymbol{U}^{T}=\left[\boldsymbol{u}_{1}, \ldots, \boldsymbol{u}_{4+k+l+m+n}\right]$. From Eqs (31) and (33), the shape functions can be derived as follow

$$
\left[\boldsymbol{N}_{1}, \ldots, \boldsymbol{N}_{4+k+l+m+n}\right]^{T}=\boldsymbol{q}^{-1} \boldsymbol{p}(\boldsymbol{\xi})
$$

The shape functions for electrical potential field $\varphi$ can be computed in a similar manner as above.

Additionally, according to Eq. (34), the slope discontinuity of the shape functions appears at extra nodes. Herein, the integration technique applied to sub-triangles [46] for cracked elements in the XFEM is used for variable-node elements.

In this study, we define refined zone artificially instead of based on posteriori error estimation. The reason is that it would be cumbersome to compute posteriori error in every step of crack growth. 
In each time step, mesh is refined around cracks by partitioning original large-scale elements. The crack element is easy to obtain by level set method. And the variable-node elements are generated accompanied with the mesh refinement. Once the small-scale elements are created, variable-node elements should be defined at the outermost layer of the refined area to work as transition between small and large elements.

\subsection{Evaluation of generalized dynamic intensity factors}

In the present work, the domain-form of the contour interaction integral is used to accurately calculate the generalized dynamic intensity factors in the piezoelectric materials, which can be found in [4] for details. The path independent electro-mechanical $J$-integral for a cracked piezoelectric materials is given by

$$
J=\int_{A}\left(\sigma_{i j} u_{i, 1}+D_{j} \varphi_{, 1}-W \delta_{1 j}\right) q_{, j} d A-\int_{A}\left(\rho \ddot{u} u_{i, 1}\right) q d A
$$

where $W=\left(\sigma_{i j} \varepsilon_{i j}-D_{j} E_{j}\right) / 2$ is the electric enthalpy density; $A$ is the area inside an arbitrary contour enclosing the crack-tip and $q$ is a smooth weight function, which has a value of unity at the crack-tip, zero along the boundary of the domain $A$, and a smooth linear variation in-between.

Let us now consider two independent dynamic equilibrium states of a piezoelectric cracked body. The first state $\left(\sigma_{i j}^{(1)}, \varepsilon_{i j}^{(1)}, u_{j}^{(1)}, D_{j}^{(1)}, E_{j}^{(1)}\right)$ corresponds to the actual state, whereas the second one $\left(\sigma_{i j}^{(2)}, \varepsilon_{i j}^{(2)}, u_{j}^{(2)}, D_{j}^{(2)}, E_{j}^{(2)}\right)$ is an auxiliary state. The interaction integral for the two states is obtained by superposition of these two states which leads to another dynamic equilibrium state.

$$
I^{(1,2)}=\int_{A}\left(\sigma_{i j}^{(1)} u_{, 1}^{(2)}+\sigma_{i j}^{(2)} u_{, 1}^{(1)}+D_{j}^{(1)} \varphi_{, 1}^{(2)}+D_{j}^{(2)} \varphi_{, 1}^{(1)}-W^{(1,2)} \delta_{1 j}\right) q_{, j} d A+\int_{A} \rho \ddot{u}^{(1)} u_{i, 1}^{(2)} q d A
$$

where

$$
W^{(1,2)}=\frac{1}{2}\left(\sigma_{i j}^{(1)} \varepsilon_{i j}^{(2)}+\sigma_{i j}^{(2)} \varepsilon_{i j}^{(1)}-D_{j}^{(2)} E_{j}^{(1)}-D_{j}^{(1)} E_{j}^{2}\right) .
$$

For linear piezoelectric solids, the electro-mechanical $J$-integral is equal to the energy release rate. Applying $J$-integral to the two states (1) and (2) and then the interaction integral $I^{(1,2)}$ can be rewritten as 


$$
\begin{aligned}
& I^{(1,2)}=K_{I I}^{(1)} K_{I I}^{(2)} Y_{11}+K_{I}^{(1)} K_{I}^{(2)} Y_{22}+K_{I V}^{(1)} K_{I V}^{(2)} Y_{33}+\left(K_{I}^{(1)} K_{I I}^{(2)}+K_{I}^{(2)} K_{I I}^{(1)}\right) Y_{12}+ \\
& \left(K_{I I}^{(1)} K_{I V}^{(2)}+K_{I I}^{(2)} K_{I V}^{(1)}\right) Y_{13}+\left(K_{I}^{(1)} K_{I V}^{(2)}+K_{I}^{(2)} K_{I V}^{(1)}\right) Y_{23}
\end{aligned}
$$

By choosing the auxiliary state 2 as the crack opening mode with $K_{I}^{(2)}=1 ; K_{I I}^{(2)}=K_{I V}^{(2)}=0$, then the interaction integral is obtained as

$$
I^{(1, I)}=K_{I}^{(1)} Y_{22}+K_{I I}^{(1)} Y_{12}+K_{I V}^{(1)} Y_{23}
$$

Similarly, other modes can be obtained as

$$
\begin{gathered}
I^{(1, I I)}=K_{I}^{(1)} Y_{12}+K_{I I}^{(1)} Y_{11}+K_{I V}^{(1)} Y_{13} \\
I^{(1, I V)}=K_{I}^{(1)} Y_{23}+K_{I I}^{(1)} Y_{13}+K_{I V}^{(1)} Y_{33}
\end{gathered}
$$

As a consequence, the generalized dynamic intensity factors are finally obtained by solving the following linear algebraic equations

$$
\left\{\begin{array}{l}
K_{I}^{(1)} \\
K_{I I}^{(1)} \\
K_{I V}^{(1)}
\end{array}\right\}=\left[\begin{array}{lll}
Y_{22} & Y_{12} & Y_{23} \\
Y_{12} & Y_{11} & Y_{13} \\
Y_{23} & Y_{13} & Y_{33}
\end{array}\right]\left\{\begin{array}{l}
I^{(1, I)} \\
I^{(1, I I)} \\
I^{(1, I V)}
\end{array}\right\}
$$

\section{Numerical results}

In this section, several benchmark numerical examples for stationary dynamic cracks in linear elastic and piezoelectric structures are presented to illustrate the accuracy and efficiency of the elaborated V-XFEM. The accuracy and efficiency is numerically confirmed through the comparison of the present approach with analytical solutions and the standard XFEM. Plane-strain condition and the impermeable crack-face boundary condition are assumed throughout the study.

\subsection{Dynamic fracture of edge crack in the semi-infinite domain}

The first numerical example for dynamic fracture analysis deals with a semi-infinite mode-I crack loaded by a tensile stress perpendicular to the crack-face. Due to the computational limits, a limited domain is modeled for analysis, whose geometry is sketched in Fig. 3. The dimensions of the limited plate are: length $L=10 \mathrm{~m}$, half-height $H=2 \mathrm{~m}$ and crack length $a=5 \mathrm{~m}$; The material 
parameters are: $E=210 \mathrm{GPa}, v=0.3, \rho=8000 \mathrm{~kg} / \mathrm{m}^{3}$. The top of the plate is subject to a tensile stress of $\sigma_{0}=500 \mathrm{MPa}$. To avoid the reflection when the tensile stress wave reaches the crack-tip, the valid time for the simulation is $t \leqslant 3 t_{c}=3 H / c_{d}=1.009 \times 10^{-3} \mathrm{~s}[4]$, where $c_{d}$ is the dilatational wave speed.

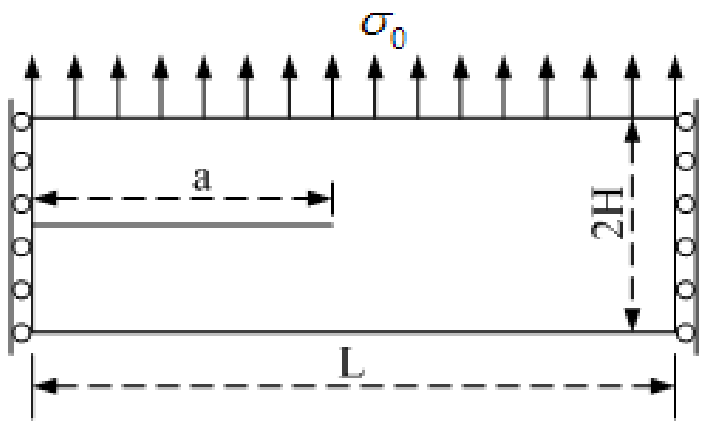

Fig. 3. Geometry of a semi-infinite mode-I crack

For convenience, the dynamic stress intensity factor (DSIF) is normalized by $\bar{K}_{I}=K_{I}^{d y n} / \sigma_{0} \sqrt{\pi a}$. Regardless of the crack propagation, the analytic solution of the mode-I DSIF can be written as [46]:

$$
K_{I}^{d y n}(0, t)= \begin{cases}0 & t<t_{c} \\ \frac{2 \sigma_{0}}{1-v} \sqrt{\frac{c_{d}\left(t-t_{c}\right)(1-2 v)}{\pi}} & t \geq t_{c}\end{cases}
$$

The relative error of normalized dynamic stress intensity factor is defined as:

$$
e r r=\left|\frac{\bar{K}_{I}-\bar{K}_{I}^{\text {analytic }}}{\bar{K}_{I}^{\text {analytic }}}\right| \times 100 \%
$$

where $\bar{K}_{I}$ and $\bar{K}_{I}^{\text {analytic }}$ are the normalized dynamic stress intensity factor of numerical solution and analytical solution, respectively.

\subsubsection{Validation and accuracy}

In order to validate the elaborated V-XFEM and demonstrate its performance in modeling dynamic fracture problems, Fig. 4 and Fig. 5 present, respectively, the comparative study of the normalized mode-I DSIFs and their percentage errors with the standard XFEM and the analytical solution. In variable-node XFEM, the original coarse mesh use $65 \times 25$ elements as shown in Fig. 6(a), and the local mesh refinement with $3 \times 3$ small scale elements are generated in where crack 
locates (see Fig. 6(b)), the total number of degree of freedom (Dof) is 6812. For comparison, the standard XFEM with uniform structured meshes of $65 \times 25$ (Dof is 3592), $79 \times 41$ (Dof is 6908) and $195 \times 75$ (Dof is 30212) elements are considered, in which the small mesh of $195 \times 75$ elements (see Fig. 6(c)) is the same as variable-node XFEM local refined mesh in the vicinity of crack.

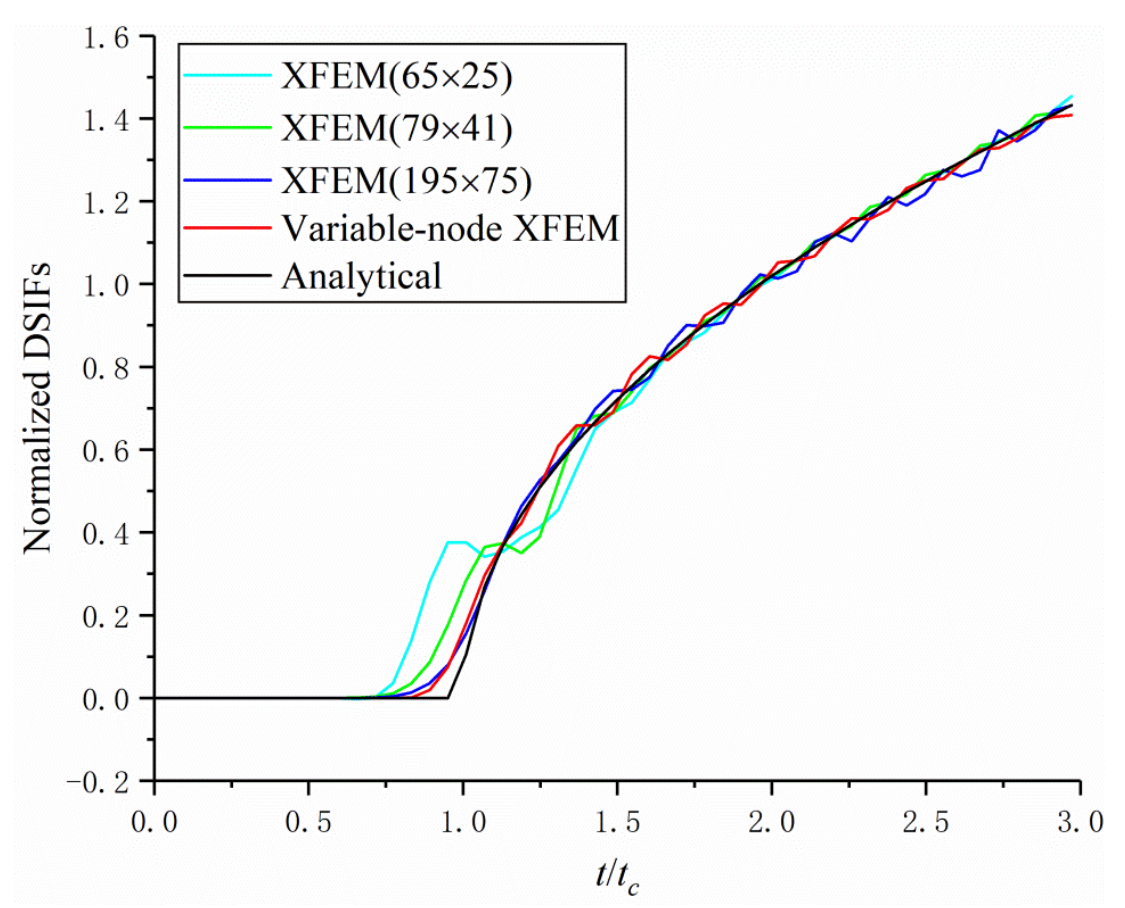

Fig. 4. Comparison of the normalized mode-I DSIFs among different methods.

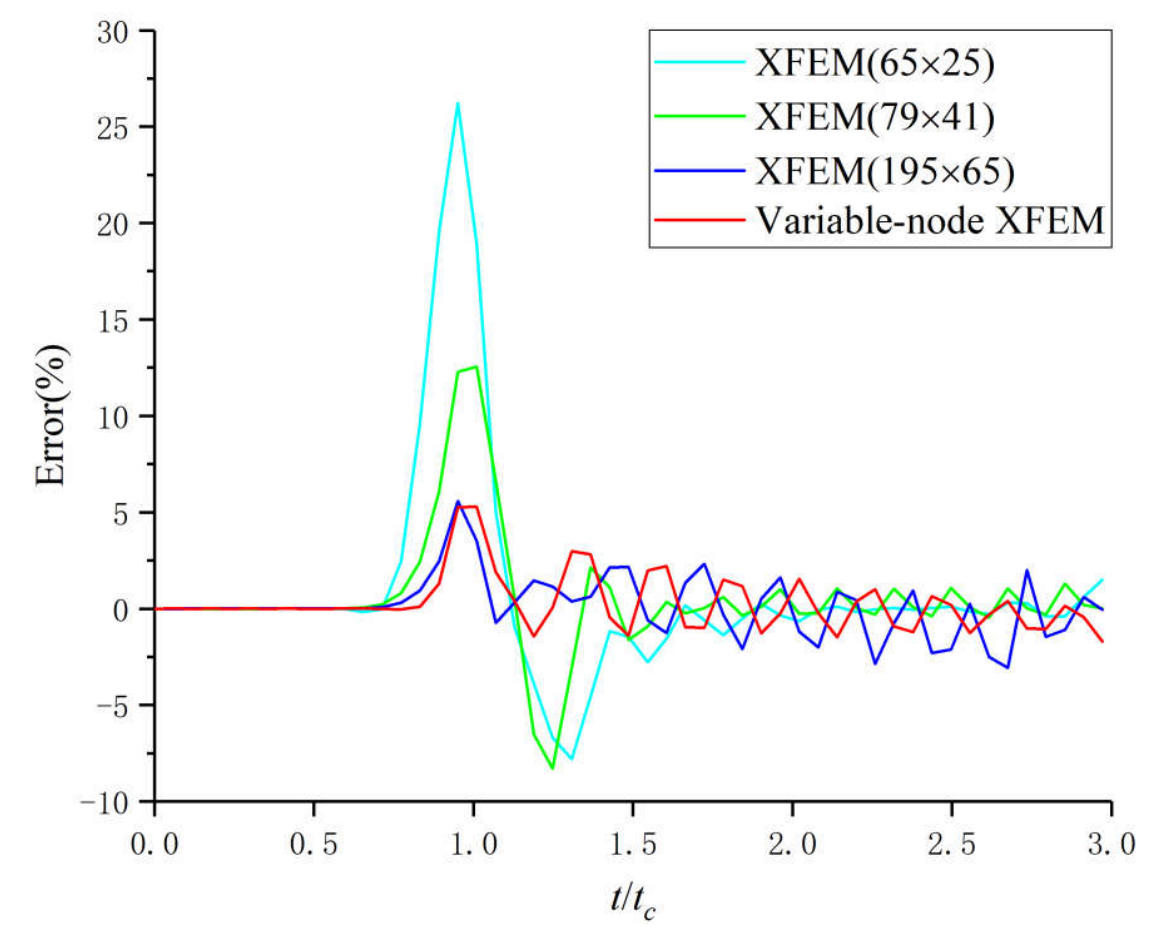

Fig. 5. Comparison of percentage errors for the normalized mode-I DSIFs among different methods. 


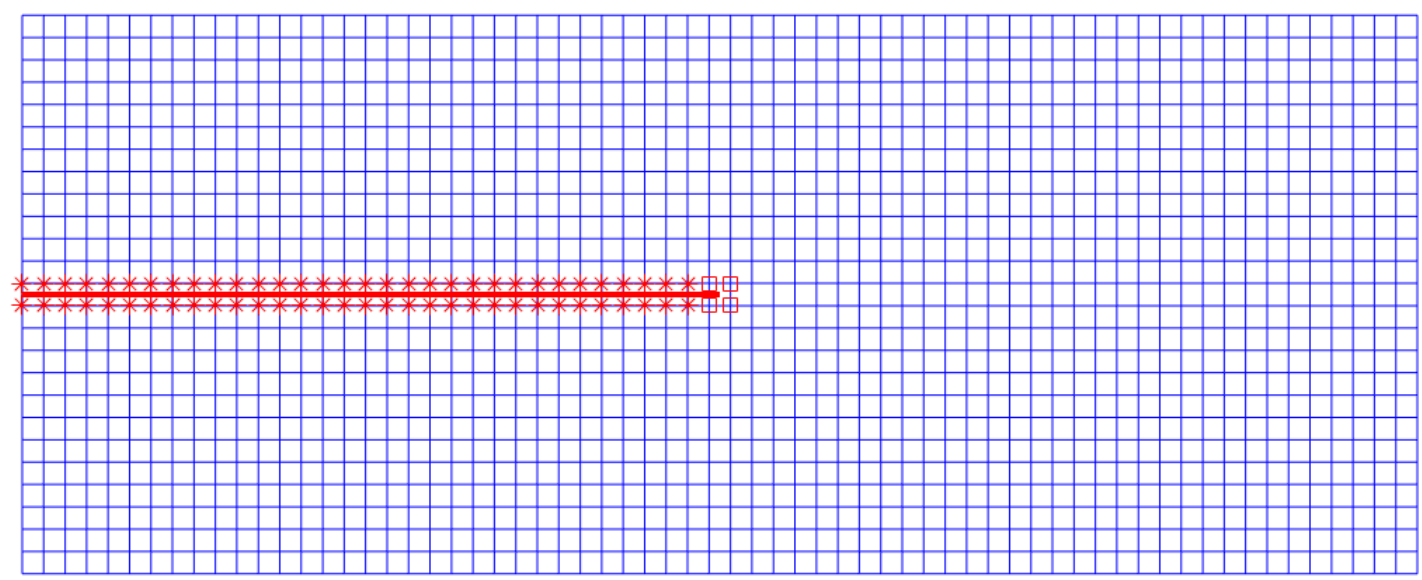

(a)

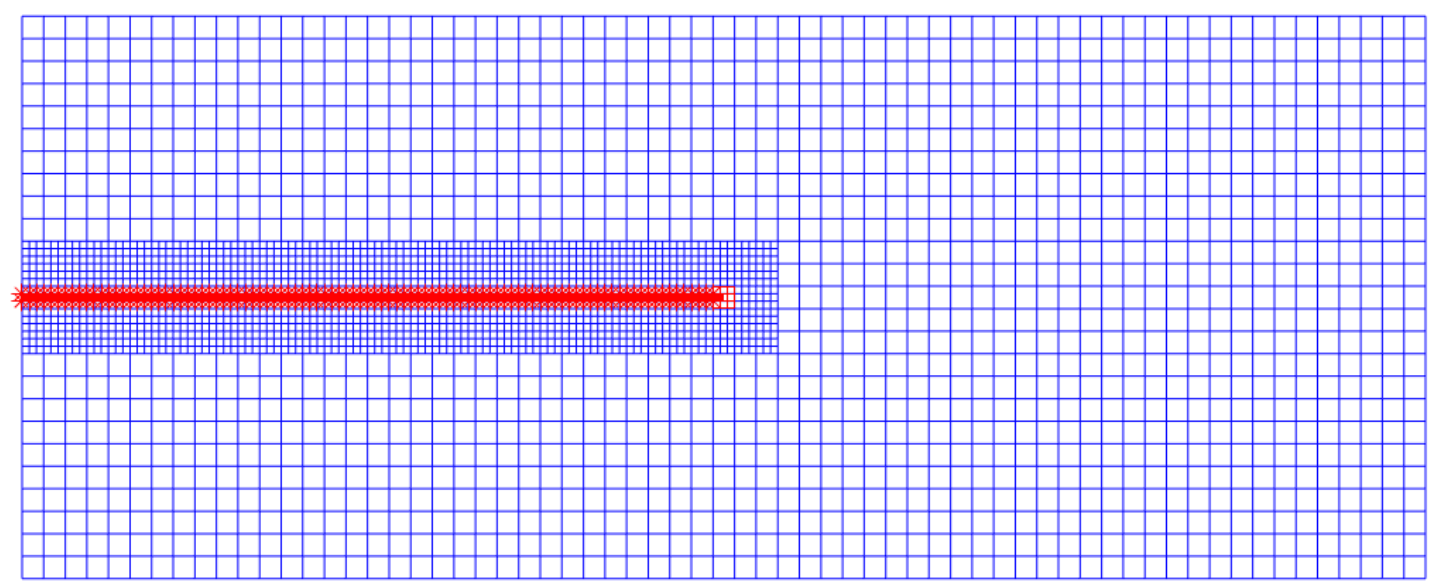

(b)

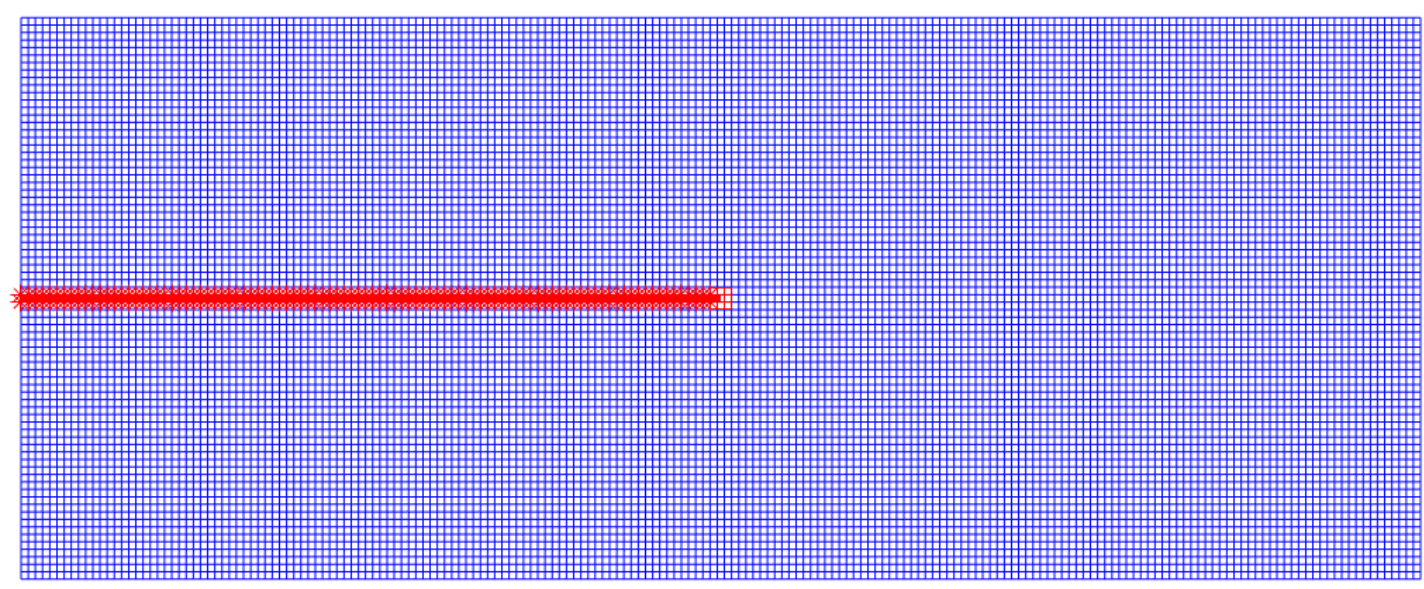

(c)

Fig. 6. Mesh of edge crack in the semi-infinite domain:(a) original coarse mesh, (b) variable-node local refined mesh, (c) standard small scale mesh.

As shown in Figs. 4 and 5, the normalized values of the DSIFs achieved by the V-XFEM match well to the analytical solution and the standard XFEM with the small mesh of $195 \times 75$ 
elements (Dof is 30212). Nevertheless, the V-XFEM with 6812 Dofs has a higher accuracy than standard XFEM with 3592 Dofs $(65 \times 25$ elements) and 6908 Dofs $(79 \times 41$ elements $)$. It can be concluded that the V-XFEM has a higher accuracy than standard XFEM.

\subsubsection{Numerical efficiency}

For comparison of the numerical efficiency, Table 1 presents a comparative study of the CPU-time consumed for three standard XFEM meshes and the variable-node XFEM mesh. As can be seen in Table 1 that the variable-node XFEM (see Fig. 6(b)) needs less computational cost than the standard XFEM with fine mesh (see Fig. 6(c)).

Table 1. Comparison of numerical efficiency between the variable-node XFEM and standard XFEM.

\begin{tabular}{ccccc}
\hline Methods & Nodes & Elements & Dofs & CPU Time(s) \\
\hline & 1716 & 1625 & 3592 & 36.4263 \\
XFEM & 3360 & 3239 & 6908 & 48.7061 \\
& 14896 & 14625 & 30212 & 179.4868 \\
\hline $\begin{array}{c}\text { Variable-node } \\
\text { XFEM }\end{array}$ & 3196 & 3025 & 6812 & 72.1416 \\
\hline
\end{tabular}

\subsection{Dynamic fracture of the bevel edge crack plate}

In order to prove the applicability and reliability of the V-XFEM for dynamic fracture problems, a mixed-mode crack is thus considered. A rectangular plate with an inclined edge crack as depicted in Fig. 7 is examined. The geometry of the plate are $L=44 \mathrm{~mm}, H=32 \mathrm{~mm}, D=16 \mathrm{~mm}$, crack length $a=22.63 \mathrm{~mm}$, and the incline angle of crack $\alpha=45^{\circ}$. The material parameters are set to be modulus of elasticity $E=29.4 \mathrm{GPa}$, Poisson's ratio $v=0.286$ and the density $\rho=2450 \mathrm{~kg} / \mathrm{m}^{3}$. The left, top and bottom of plate supported in normal direction, as shown in Fig.7, the right end is subjected to a step impact loading $\sigma_{0} H(t)$, where $H(t)$ represents the Heaviside step function. The total computation time is $3 \times 10^{-5} \mathrm{~s}$ and the number of iterations is 50 times. As shown in Fig. 8(a), the variable-node mesh with $15 \times 8$ coarse elements and $3 \times 3$ small scale elements in the vicinity of crack is used for dynamic fracture problems with inclined edge crack. For validation purpose, the computed result is then compared with those obtained from standard XFEM with fine mesh of $45 \times 25$ elements as shown in Fig. 8(b). Figs. 9 shows that the normalized mixed-mode DSIFs 
achieved by the V-XFEM agree well to XFEM with a fine mesh.

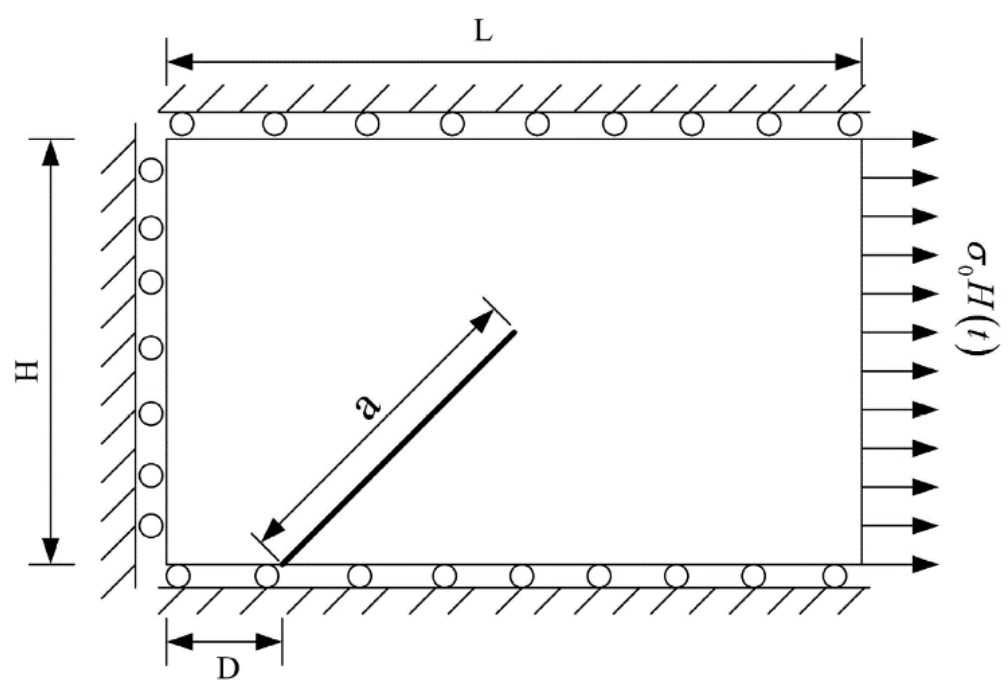

Fig. 7. Geometry of a rectangular plate with an inclined crack.

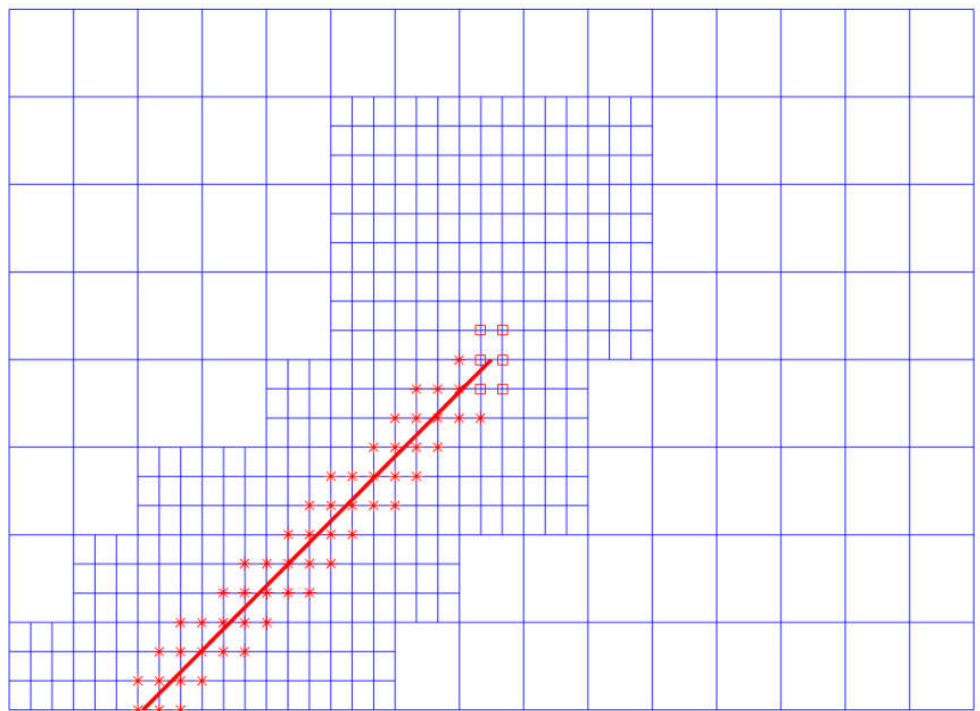

(a) 


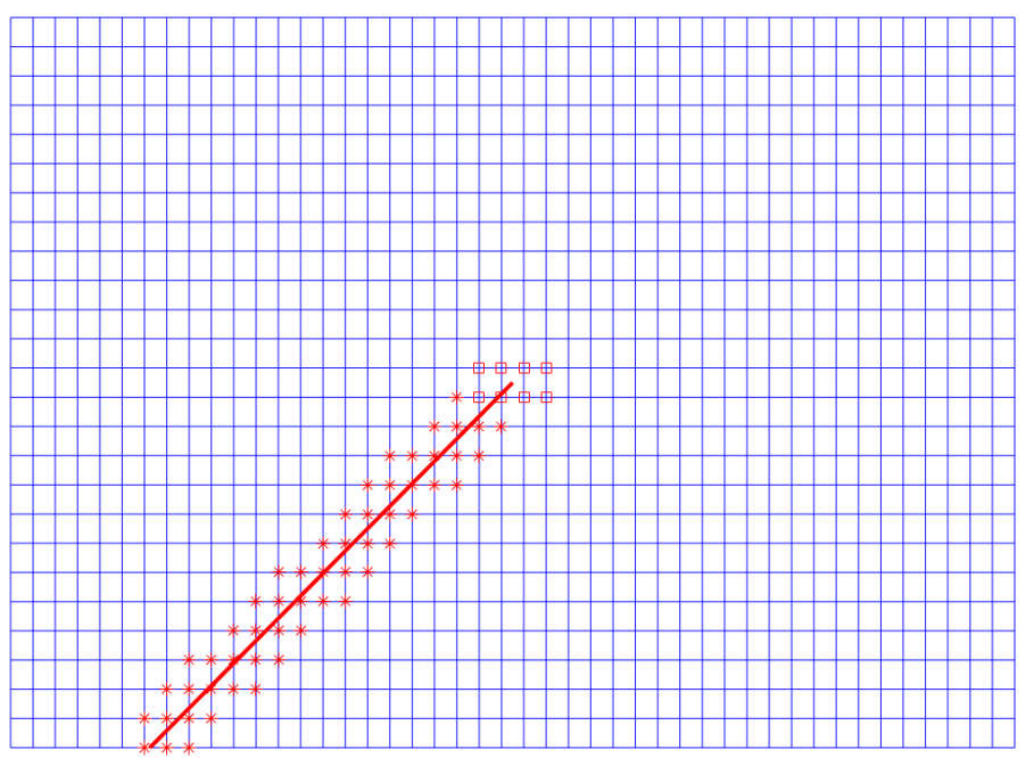

(b)

Fig. 8. Mesh of the inclined edge crack plate: (a) variable-node local refined mesh, (b) standard small scale mesh.

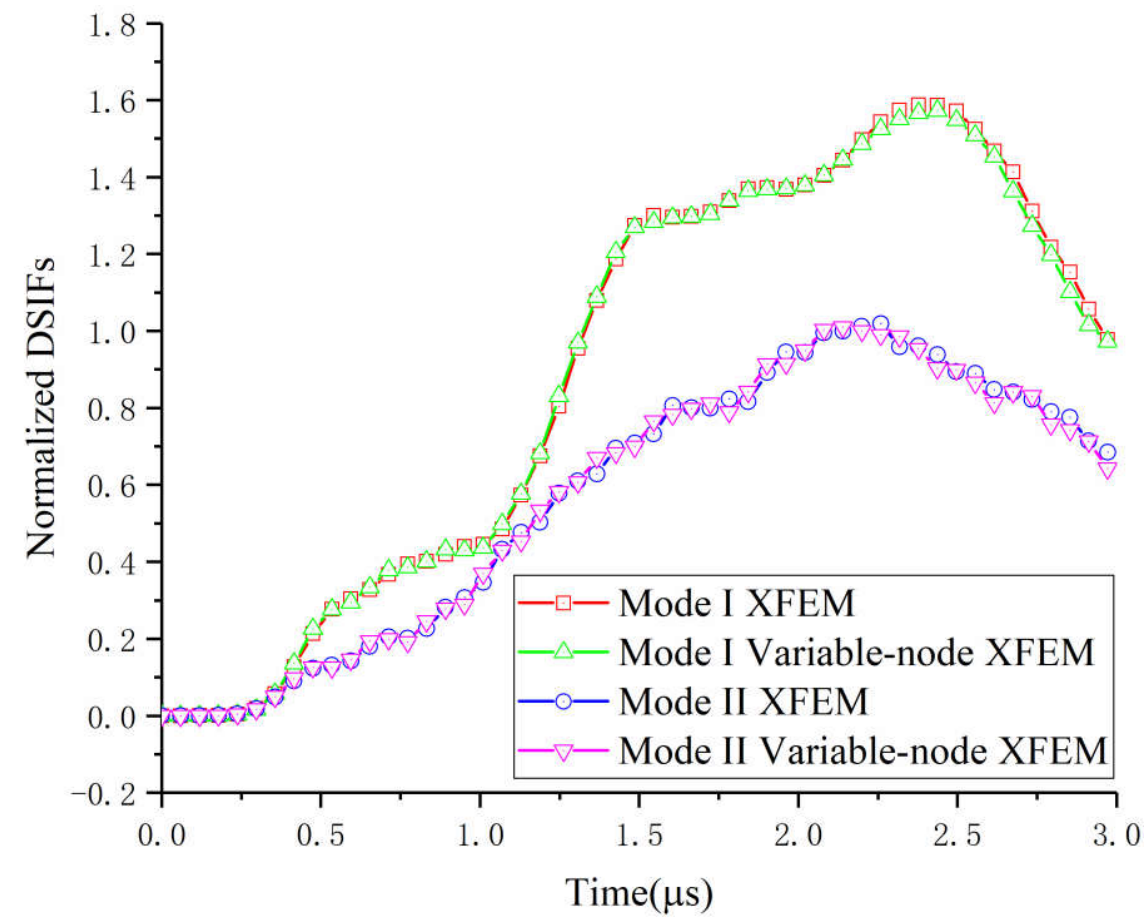

Fig. 9. Comparison of the normalized mixed-mode DSIFs for a rectangular plate with a inclined edge crack.

\subsection{Dynamic fracture of a center crack in a piezoelectric structure}

In this dynamic study, we extend the V-XFEM to piezoelectric structure. Now, we consider a PZT-5H piezoelectric plate contains a central crack of length 2a, as depicted in Fig. 10. The geometry of the plate is $h=20 \mathrm{~mm}$ and a $=2.4 \mathrm{~mm}$. The material parameters of PZT-5H are set as: 
$C_{11}=126.0 \mathrm{GPa}, \quad C_{13}=84.1 \mathrm{GPa}, \quad C_{33}=117.0 \mathrm{GPa}, C_{44}=23.0 \mathrm{GPa}, \quad e_{31}=-6.5 \mathrm{C} / \mathrm{m}^{2}$, $e_{33}=23.3 \mathrm{C} / \mathrm{m}^{2}, \quad e_{15}=17.0 \mathrm{C} / \mathrm{m}^{2}, \quad \kappa_{11}=15.04 \mathrm{C} /(\mathrm{GVm}), \quad \kappa_{33}=13.0 \mathrm{C} /(\mathrm{GVm})$, density $\rho=7500 \mathrm{~kg} / \mathrm{m}^{3}$. Both impact mechanical $\sigma(t)=\sigma_{0} H(t)$ and electrical $D(t)=\lambda k_{33} / e_{33} \sigma_{0} H(t)$ loadings are considered in the study, where $\lambda$ is electrical impact loading parameter. The total computation time is $t=5 h / c_{\mathrm{L}}$ and the time-step is $\Delta t=0.1 h / c_{\mathrm{L}}$, in which $c_{\mathrm{L}}=\sqrt{\left(C_{33}+e_{33}^{2} / k_{33}\right) / \rho}$ is the velocity of the longitudinal wave along the second principal material axis. A comparison of the normalized dynamic intensity factors with the six crack tip enrichments [36] and the standard four crack tip enrichments is presented in Fig 11. It can be seen that the results are matched very well and consistent with the conclusion of Bechet et al [36] that the four-fold enrichment is almost as efficient, concerning accuracy both in energy and in the SIFs. However, the four-fold enrichment is simpler to implement and involves less computational overhead, because it adds only four degrees of freedom (dofs) per regular dof, instead of six. Therefore, the four-fold enrichment is adopted in the rest of this paper.

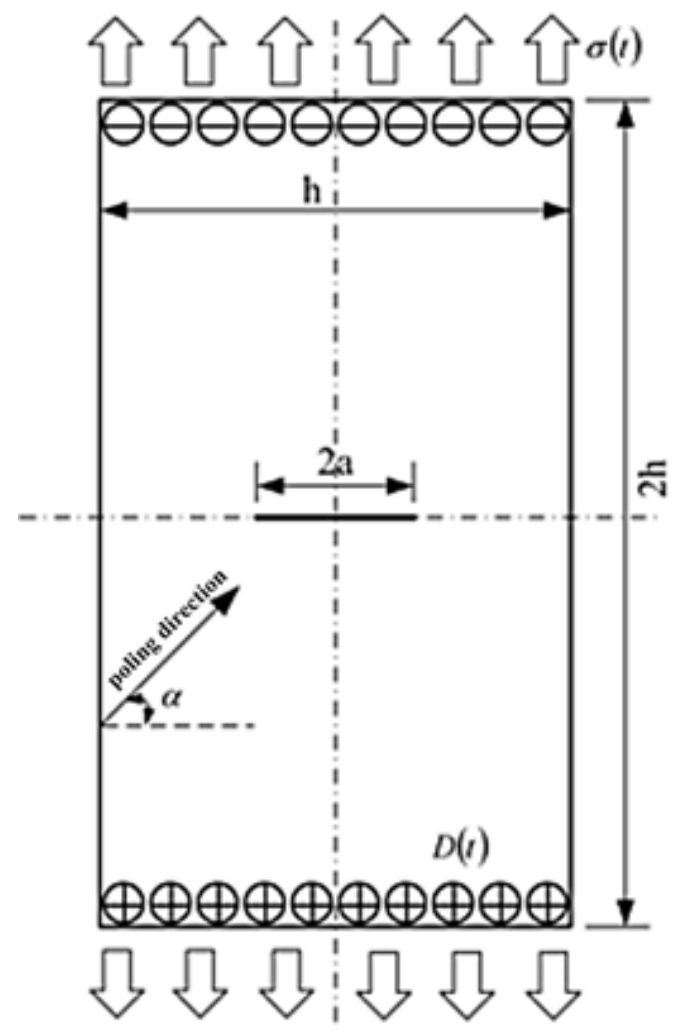

Fig. 10. A rectangular piezoelectric plate with a central crack under impact loading. 

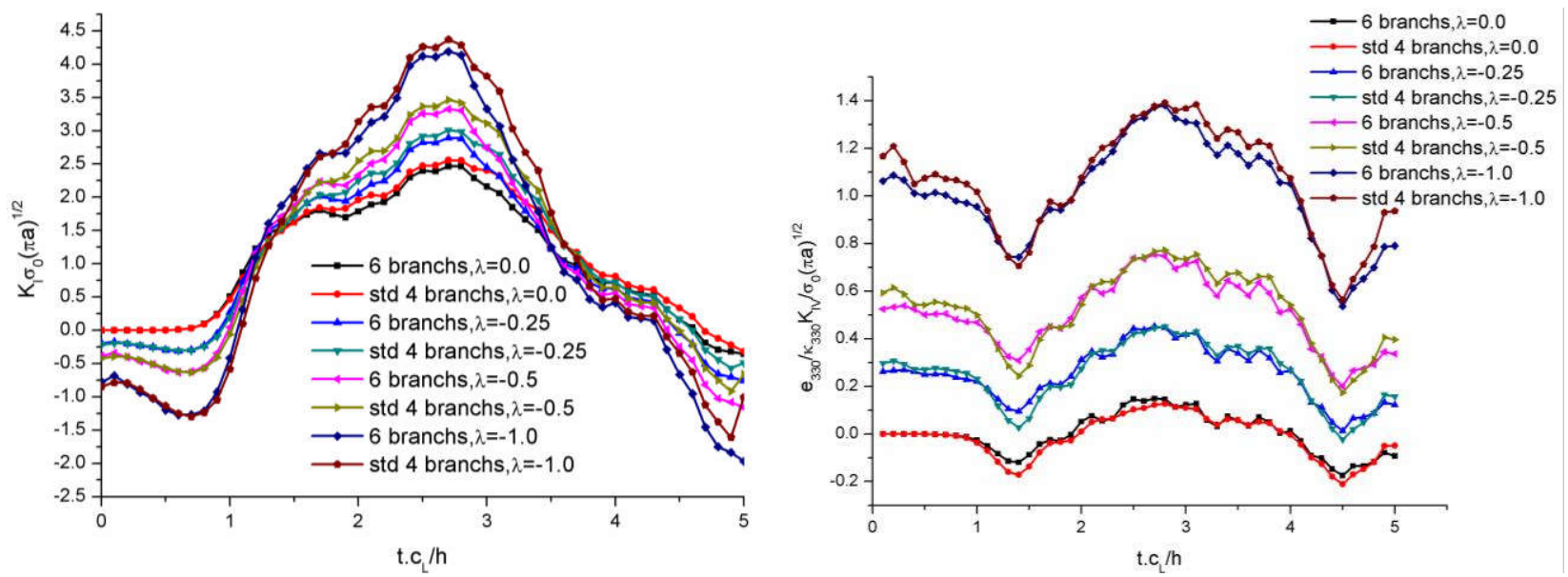

Fig. 11. Comparison of the normalized dynamic intensity factors for different crack-tip enrichment functions

The problem is solved by variable-node XFEM with $19 \times 39$ coarse elements and $3 \times 3$ small scale elements in the vicinity of crack (see in Fig. 12a), and standard XFEM with a regular fine mesh of $50 \times 100$ quadrilateral elements for comparison (see in Fig. 12b).

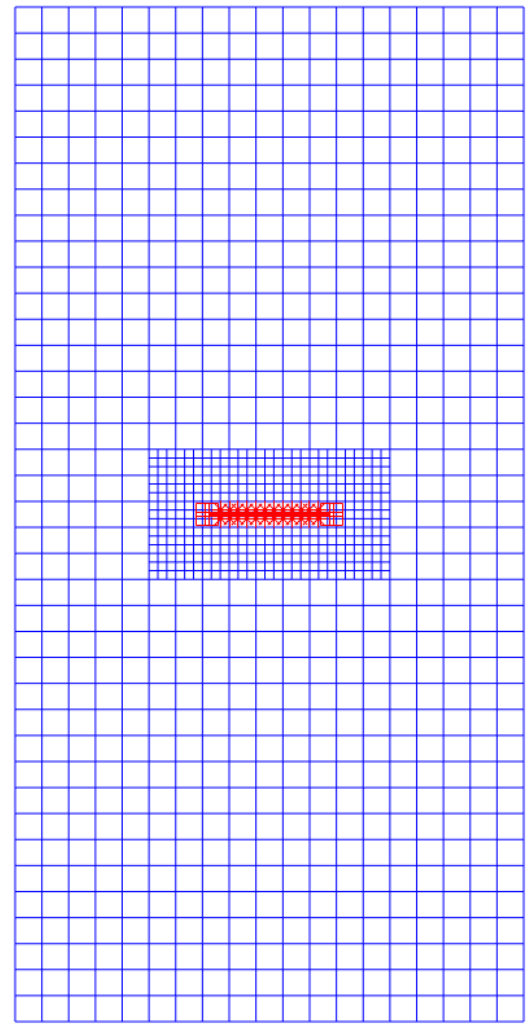

(a)

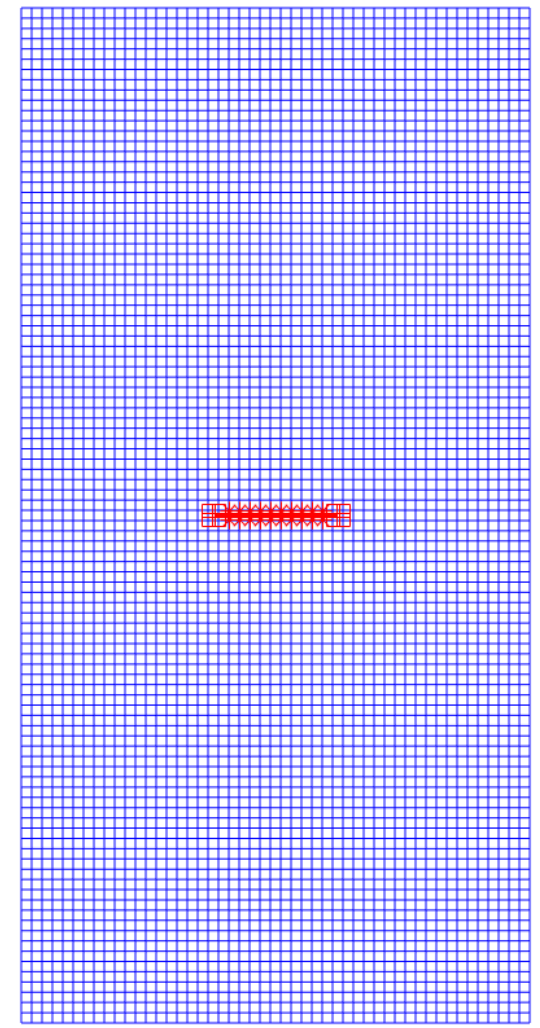

(b)

Fig. 12. Mesh of the rectangular piezoelectric plate with a central crack: (a) variable-node local refined mesh, (b) standard small scale mesh. 
We first consider the plate only subjected to an impact mechanical loading i.e. $\lambda=0$. Fig. 13 presents a comparison of the V-XFEM results for the normalized dynamic stress intensity factors $\bar{K}_{I}=K_{I}^{d y n} / \sigma_{0} \sqrt{\pi a}$ and normalized dynamic electrical displacement intensity factors $\bar{K}_{I V}=K_{I V}^{d y n} k_{33} / e_{33} \sigma_{0} \sqrt{\pi a}$ with those obtained by the standard XFEM, which shows very good agreement with each other. It also can be confirmed here that a pure mechanical impact causes an electrical field in the considered piezoelectric solids.

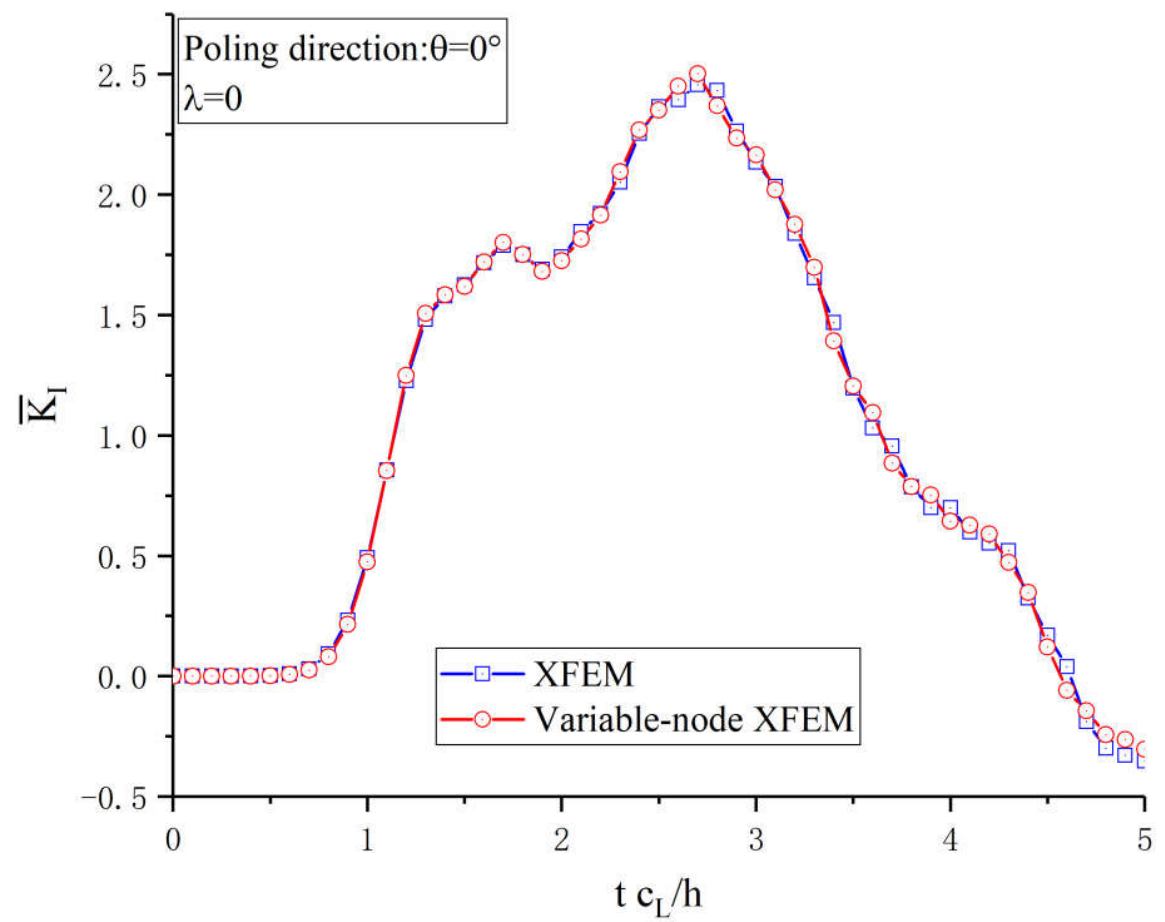

(a) 


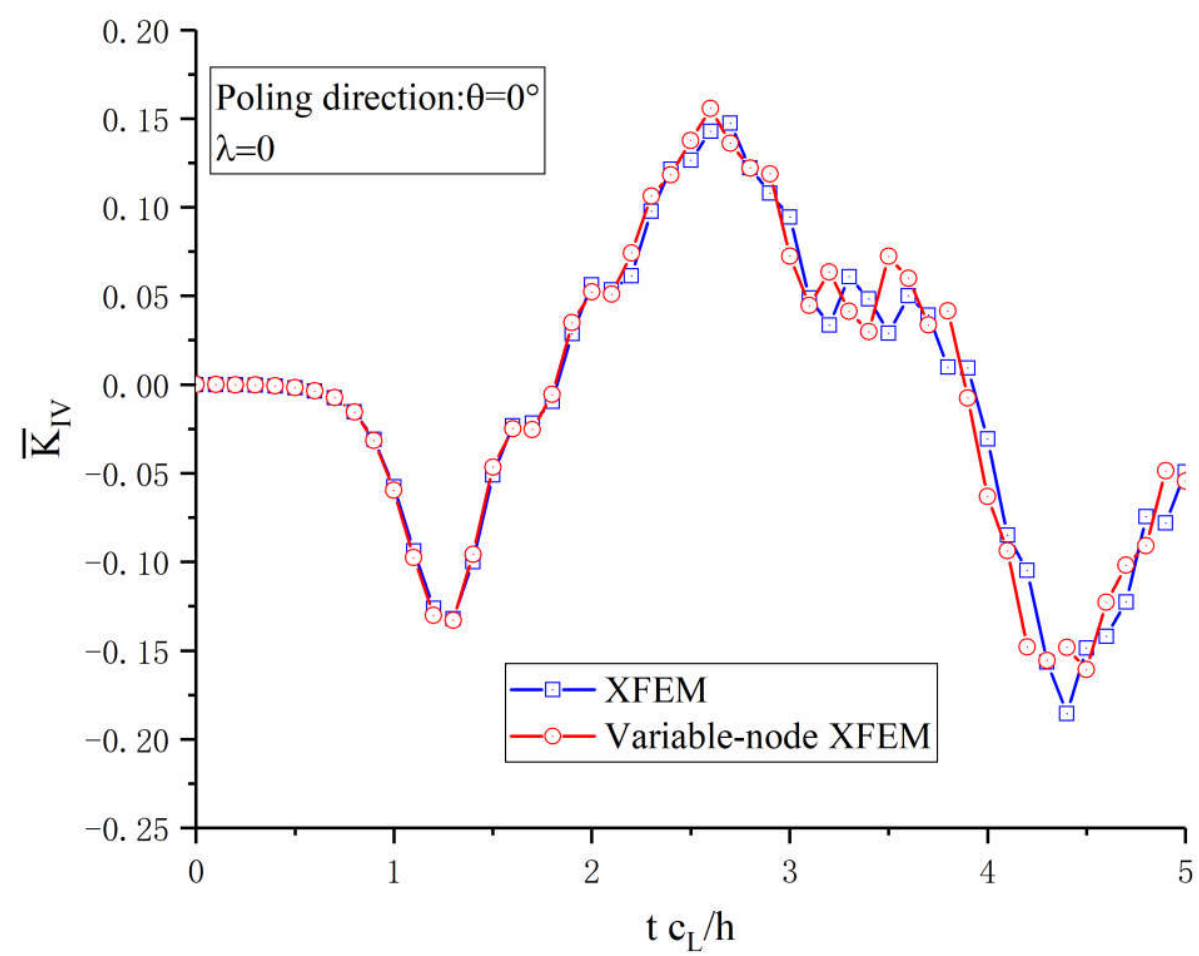

(b)

Fig. 13. A comparison of the normalized dynamic intensity factors (a) normalized dynamic stress intensity factor, (b) normalized dynamic electrical displacement intensity factor.

Now we consider the plate only subjected to an impact electrical loading. The computed results for the normalized dynamic intensity factors are presented in Fig. 14 in comparison with the standard XFEM solutions with small scale mesh. The present results match well with the standard XFEM solutions. It can be observed that the amplitude of the normalized dynamic intensity factors is the same when changing the direction in the electrical loading and the only change is their sign. It is worth noting that a pure electrical impact also induces a dynamic stress intensity factor. In Fig. 13, under a pure mechanical loading, the normalized dynamic stress intensity factors $\bar{K}_{I} \neq 0$ until the mechanical wave impinges on the crack at the time around $t=1.0$. This is because the elastic waves induced by the mechanical impact require some time to reach and open the crack. However, due to the quasi-electrostatic assumption for the electrical field, the variation of the $\bar{K}_{I}$ starts from $t=0$ under an pure electric loading. It means that the cracked plate is immediately subjected to an electrical impact and the crack thus opens at $t=0$. 


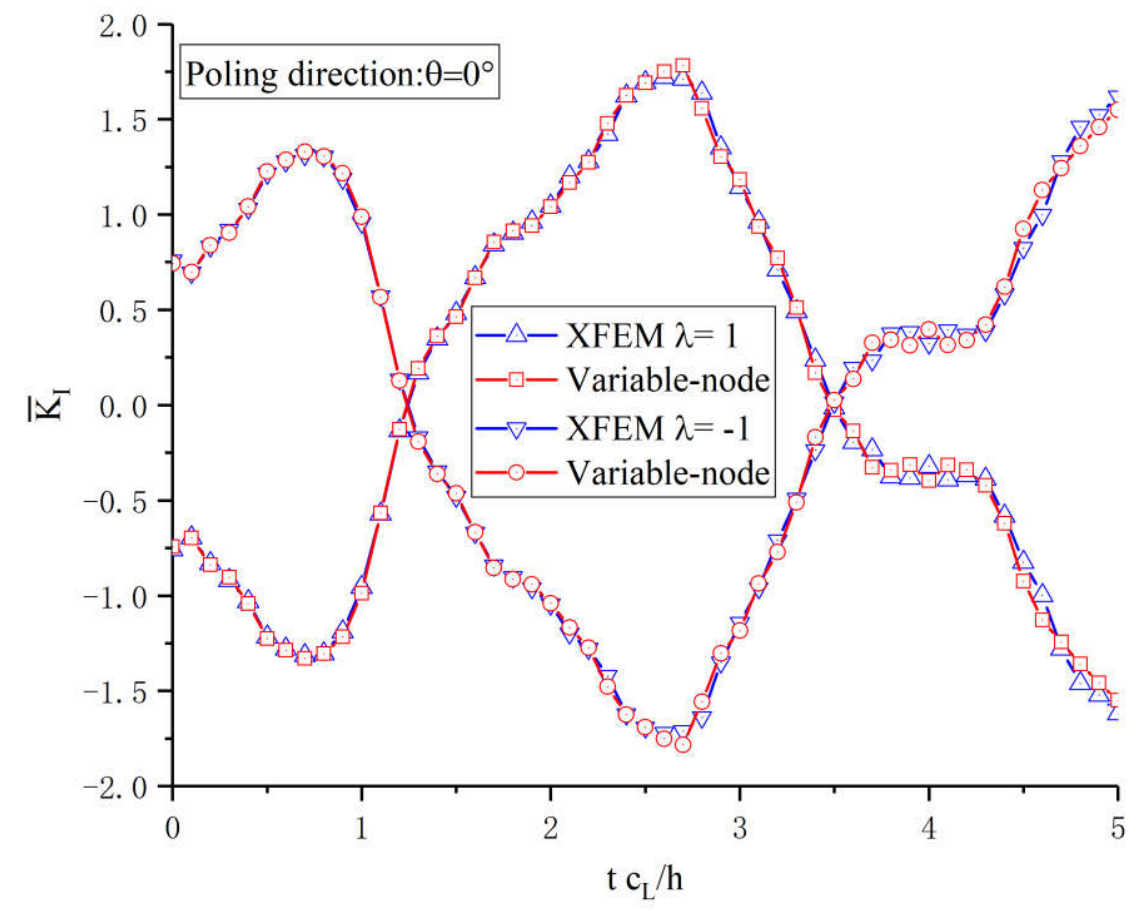

(a)

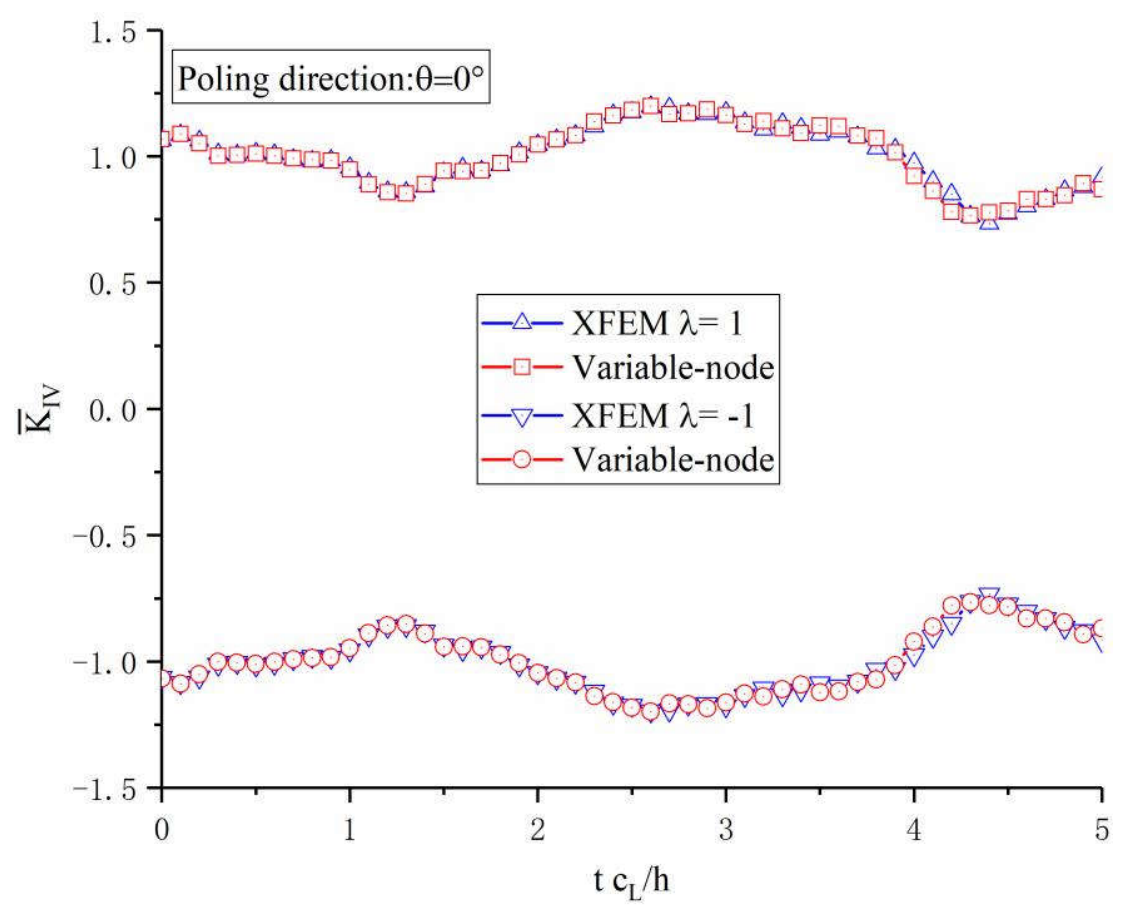

(b)

Fig. 14. A comparison of the normalized dynamic intensity factors (a) normalized dynamic stress intensity factor, (b) normalized dynamic electrical displacement intensity factor.

For the fracture behavior of piezoelectric materials, the most important and interesting issue is the investigation of the effects induced by both the mechanical and electrical loading on the 
dynamic fracture parameters. Here, we consider the same example but subjected simultaneously to a combined mechanical and electrical impact load. First, the intensity of the electrical impact $\lambda=1$ is studied and the computed results for the normalized dynamic intensity factors are shown in Fig. 15. A good agreement with each other is found, which further confirms the effective of the present variable-node XFEM. Next, we discussed the effects of the intensity of the electrical impact loading on the normalized dynamic intensity factors. The electrical loading parameter $\lambda$ is varied and the normalized dynamic intensity factors are evaluated individually and then depicted in Fig. 16. It can be seen that the maximum values of the normalized dynamic intensity factors are reduced as increase $\lambda$ and the electrical impact affects the normalized dynamic stress intensity factors significantly. It also can be concluded that the normalized dynamic electrical displacement intensity factor seems weakly dependent on the time but has a strong dependence on the load parameter $\lambda$, which is also a consequence of the quasi-electrostatic assumption of the electrical field.

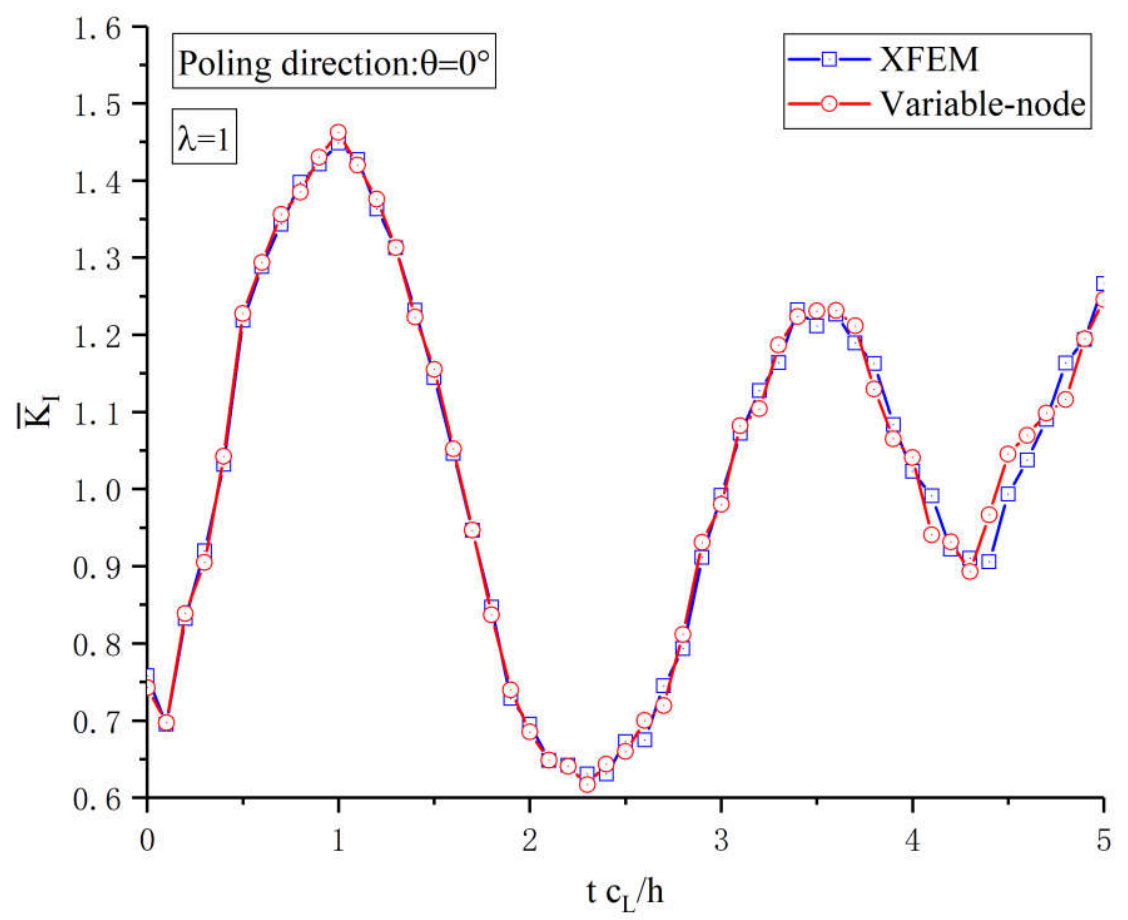

(a) 


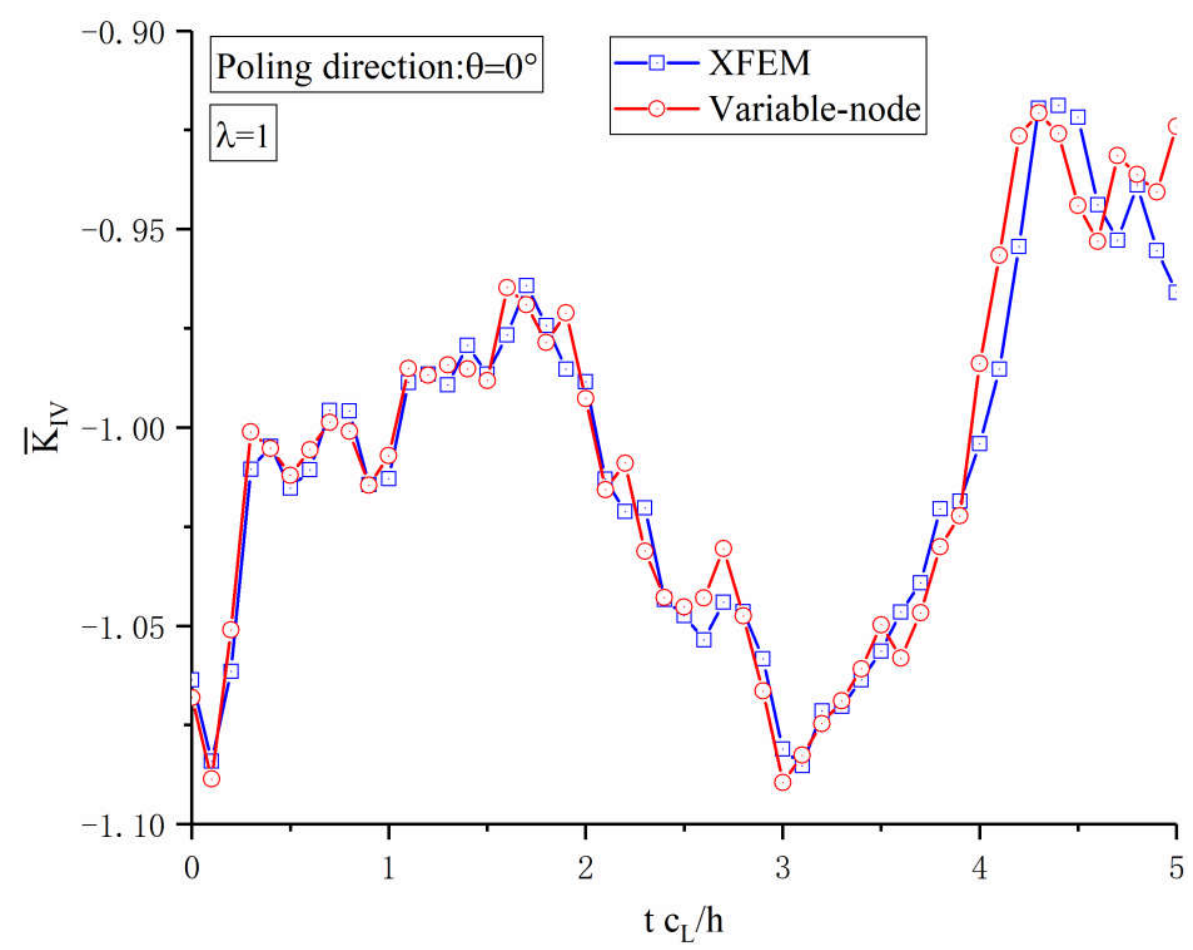

(b)

Fig. 15. A comparison of the normalized dynamic intensity factors (a) normalized dynamic stress intensity factor,

(b) normalized dynamic electrical displacement intensity factor.

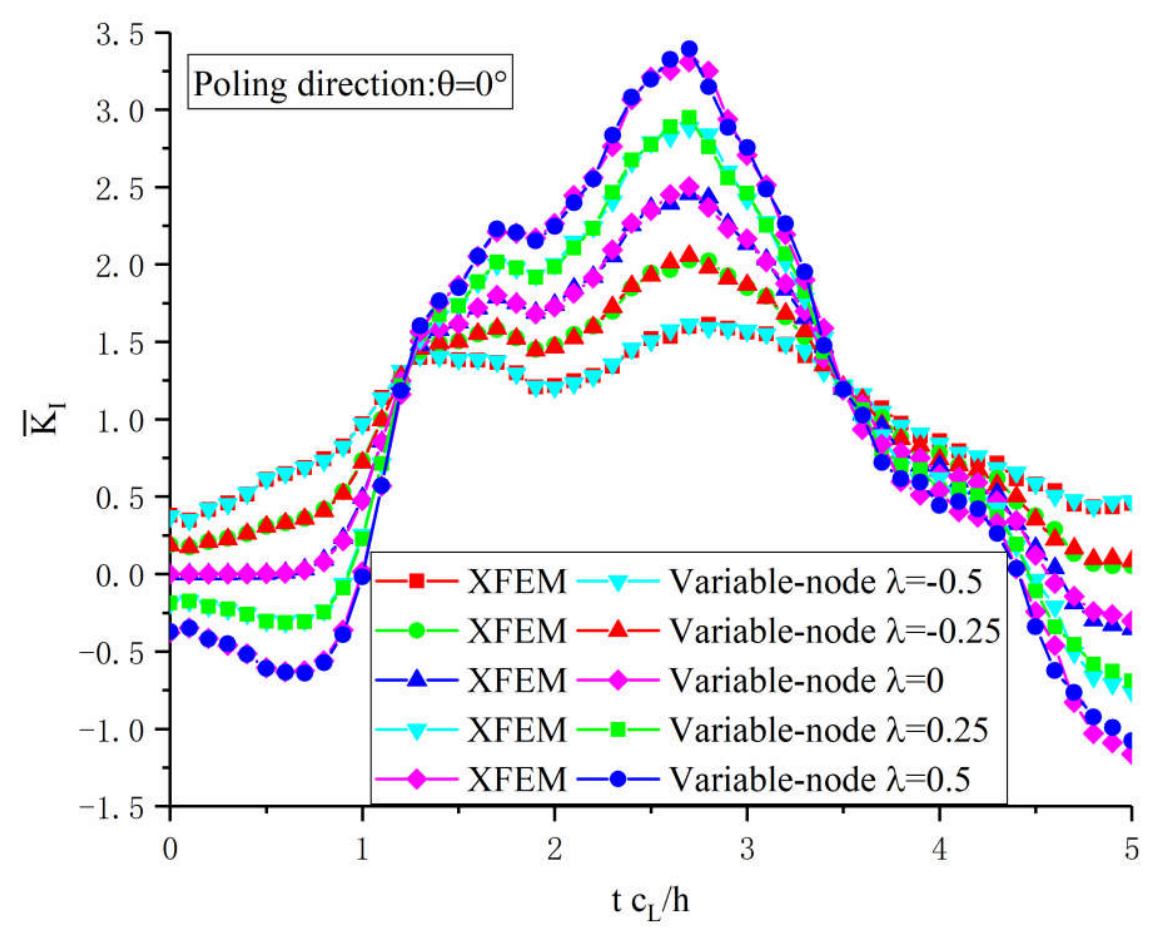

(a) 


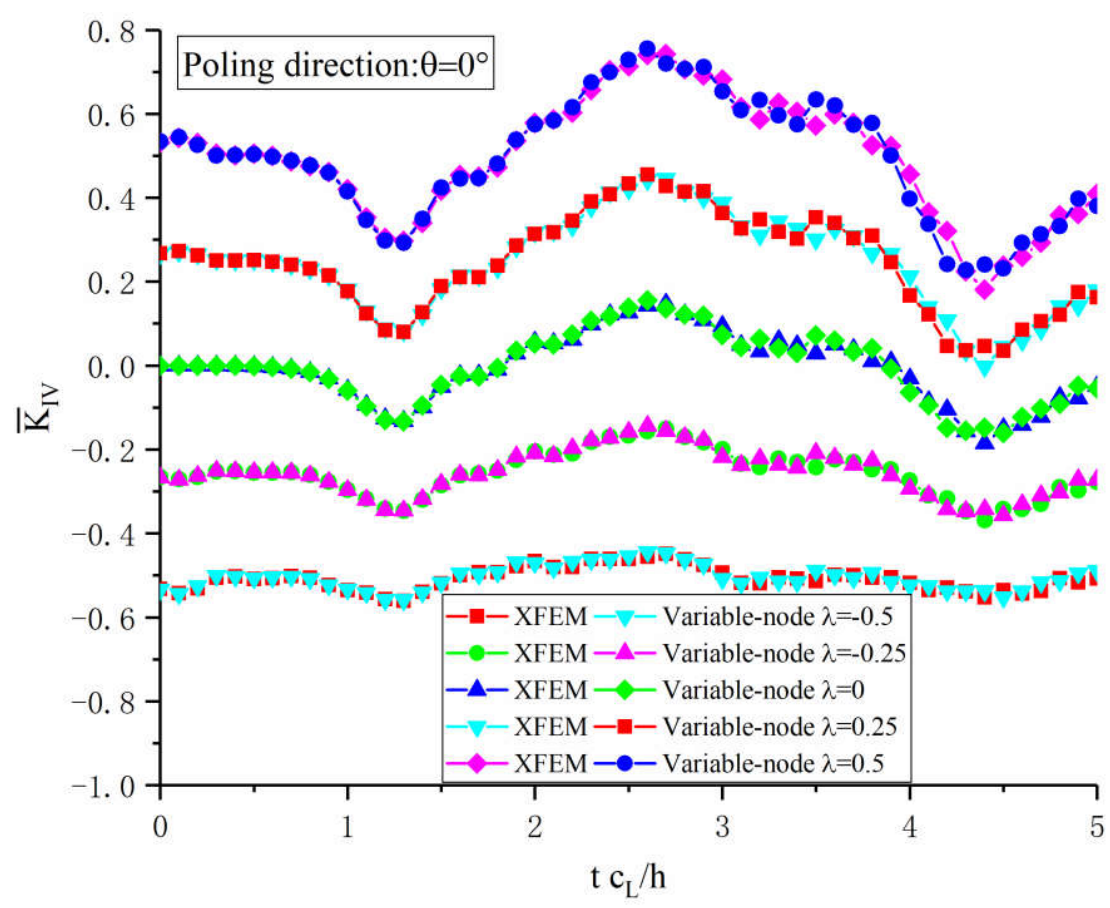

(b)

Fig. 16. Normalized dynamic intensity factor (a) normalized dynamic stress intensity factor, (b) normalized dynamic electrical displacement intensity factor.

Finally, the influence of the orientation of the material poling direction on the normalized dynamic intensity factor is discussed here. Fig. 17 shows a comparison of the normalized dynamic intensity factors derived from both methods for different poling direction. It can be observed in Fig. 17 that the normalized dynamic intensity factors have a significant dependence on the poling angle $\theta$. Beside, the electrical displacement intensity factors increase as the poling angle increase, and they are equal to zero when $\theta=90^{\circ}$, due to the piezoelectric effect vanishes for a crack parallel to the poling direction. 


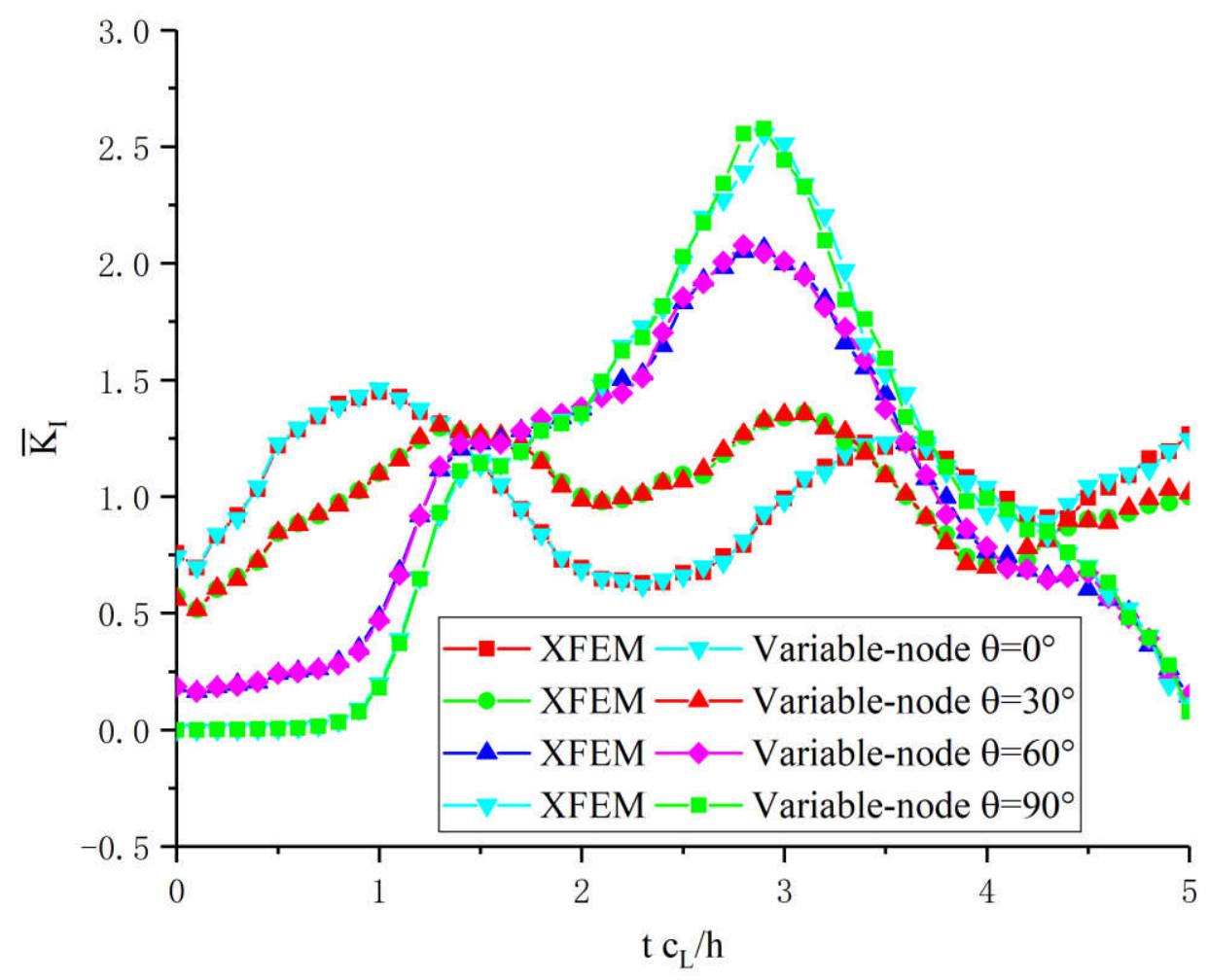

(a)

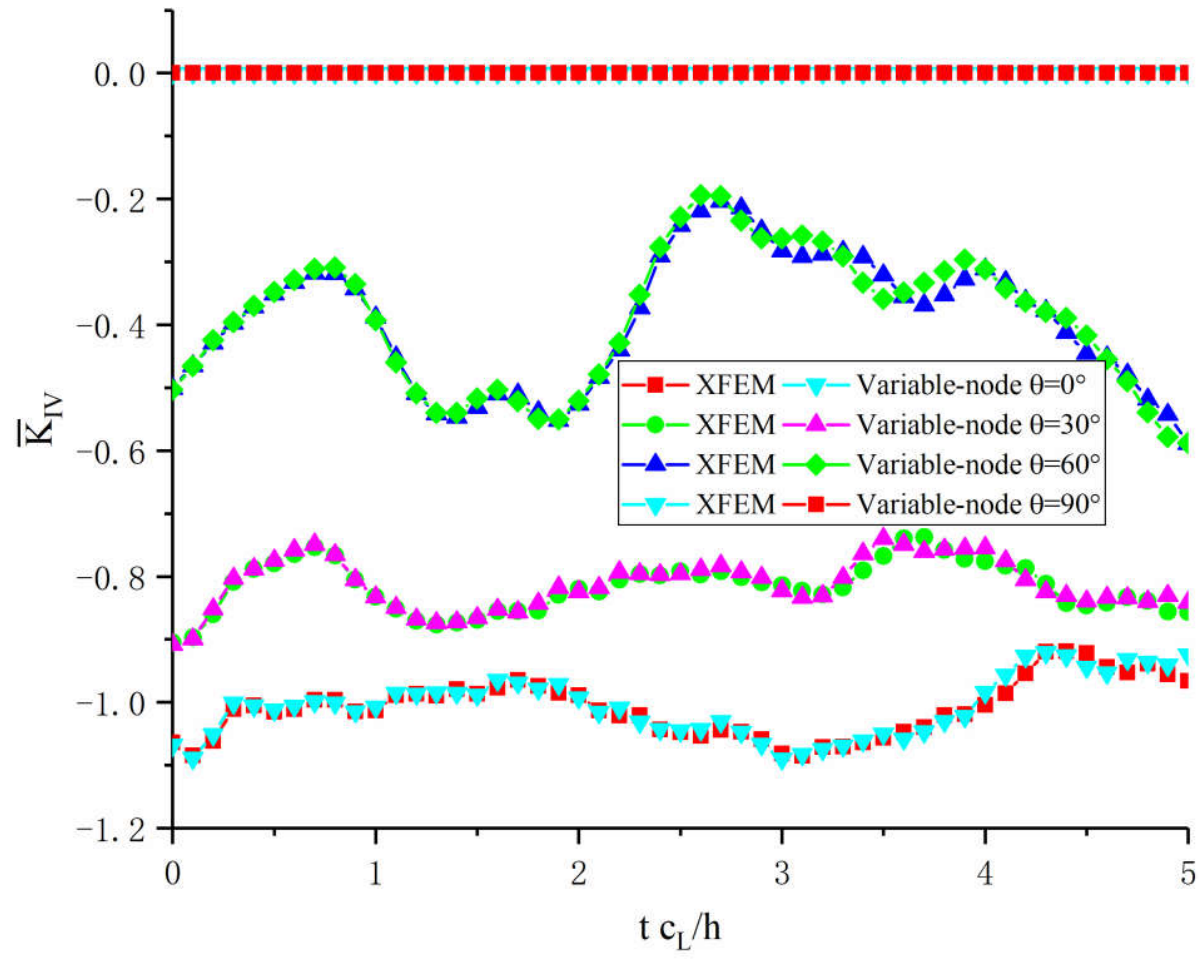

(b)

Fig. 17. Normalized dynamic intensity factor for different poling direction (a) normalized dynamic stress intensity factor, (b) normalized dynamic electrical displacement intensity factor. 


\subsection{Crack propagation simulation in piezoelectric materials}

The last numerical example studied a homogeneous finite 2-D rectangular piezoelectric plate with size $H=2 \mathrm{~mm}$ having an edge crack for crack propagation under electro-mechanical loading. The initial crack length $\mathrm{a}=0.4 \mathrm{~mm}$ has been considered for simulation. The piezoelectric domain along with boundary conditions prescribed on the plate has been indicated in Fig. 18. The former

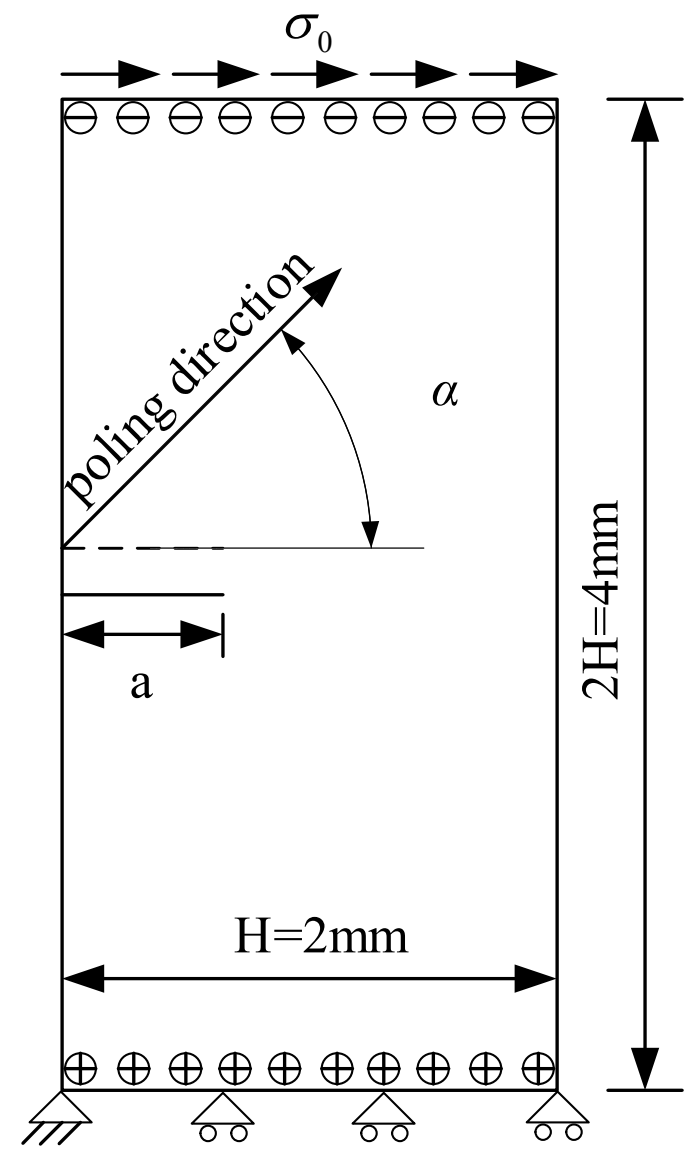

Fig. 18. Piezoelectric Plate with an edge crack.

piezoelectric material PZT-5H is taken for this study and the poling angle $\theta=90^{\circ}$. Both mechanical and electrical loadings are considered in the study. Although there are lot of crack growth criteria [48, 49] available to determine the direction of crack propagation in structural materials, appropriate exact crack growth criteria for piezoelectric solids are still being developed due to their complicated inherent coupled electro-mechanical characteristics and material anisotropic features. As the main aim of this paper is to implement V-XFEM approach for modeling crack growth for piezoelectric solids, the modified hoop SIF criterion [50] is utilized as the fracture 
criterion in order to predict crack growth direction. The local refined area is obtained by extending 3 layers from initial elements where crack locates and then $3 \times 3$ small scale elements are generated in the refined zone. The crack increment length is set to be 0.12 which is about twice the initial element size. The crack growth profiles for the first, second, fourth and the fifth crack increment steps which corresponds to critical crack length is presented in Fig. 19. It can be see that the V-XFEM can effectively simulate crack propagation.

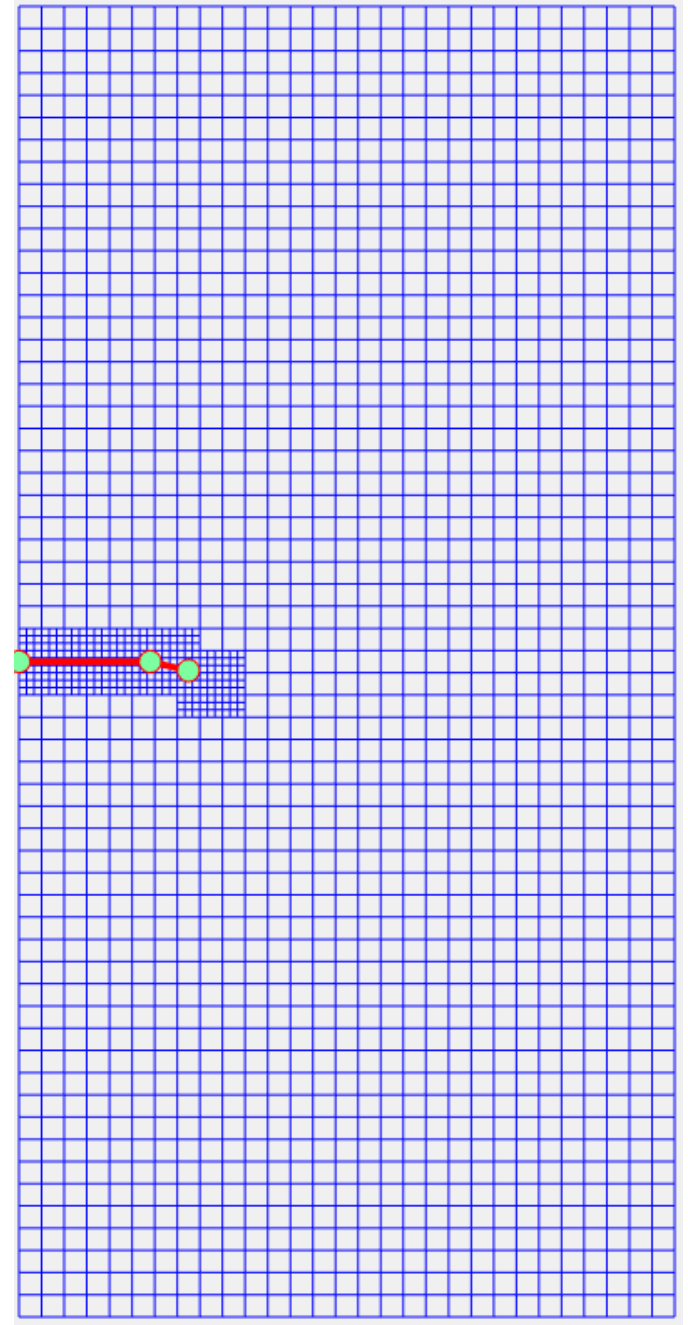

(a) The first crack increment step.

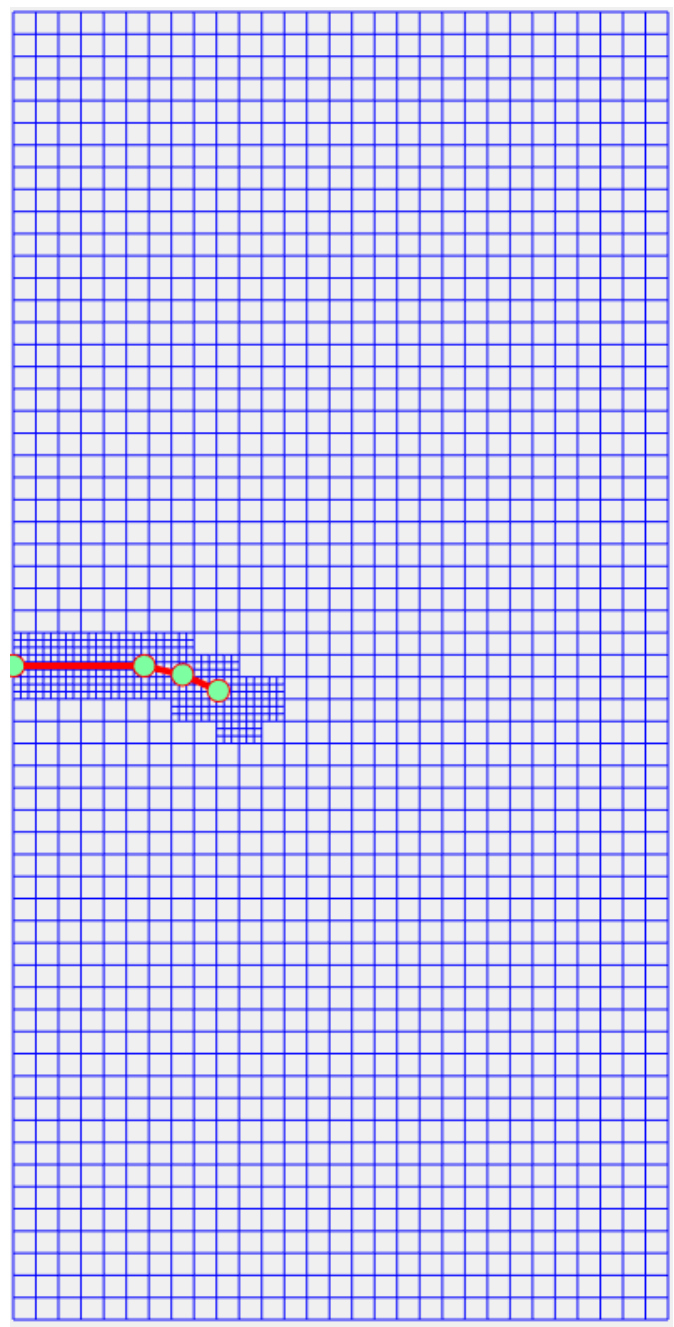

(b) The second crack increment step. 


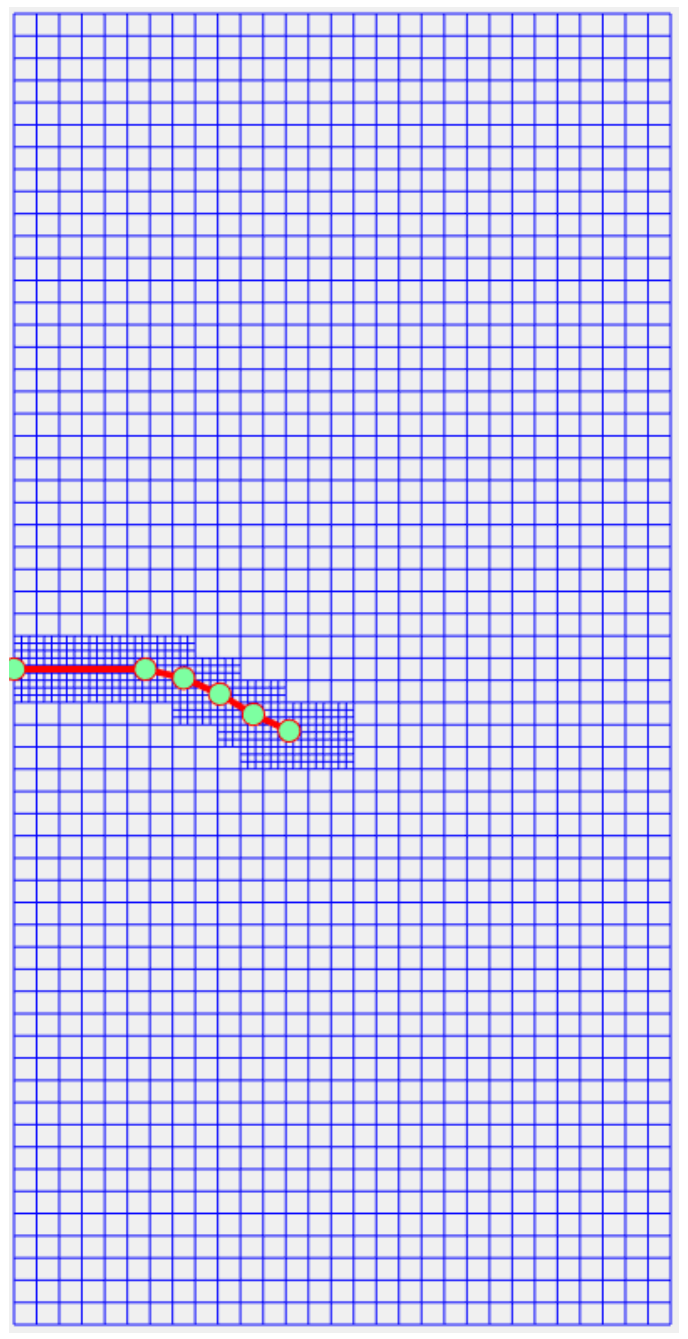

(c) The fourth crack increment step.

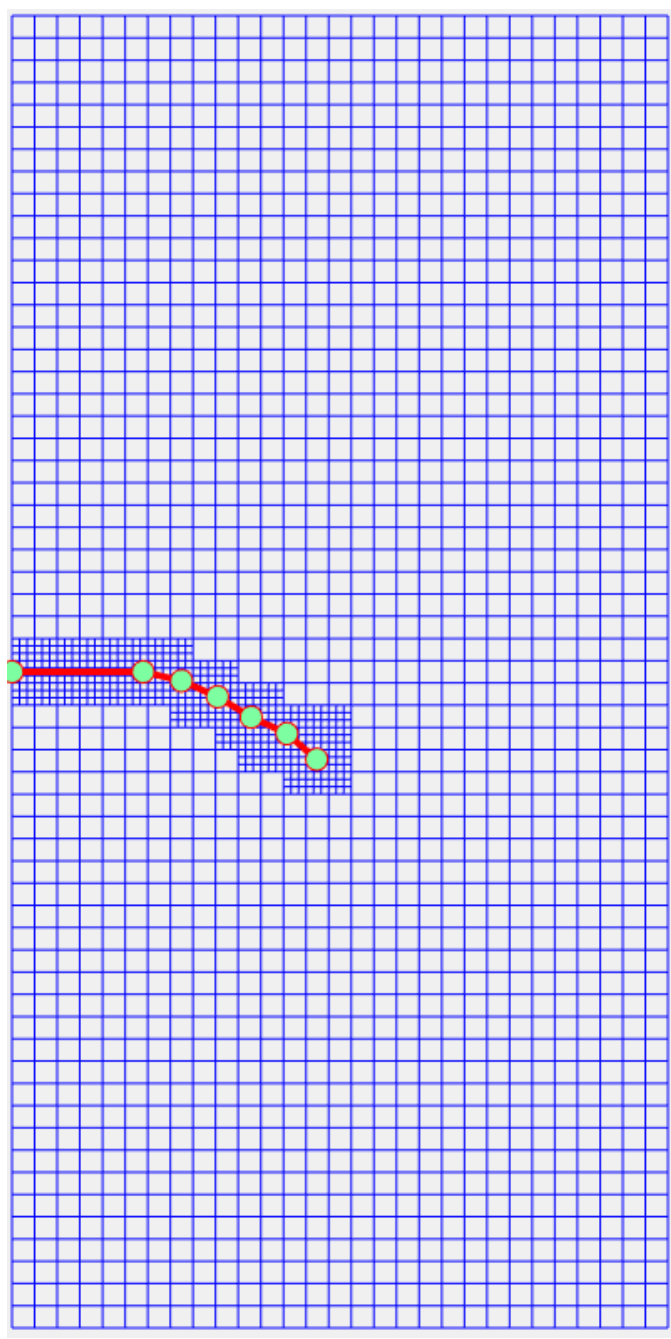

(d) The fifth crack increment step.

Fig. 19. The crack growth profiles for the first, second, fourth and the fifth crack increment steps

\section{Conclusions}

This paper elaborates the V-XFEM to simulate dynamic fracture problems subjected to impact loads for linearly uncoupled and coupled phenomena. The linearly elastic and piezoelectric problems are considered as illustrated examples in order to show the efficiency and accuracy of V-XFEM. A dynamic XFEM integrated with variable-node elements as well as the implicit time integration scheme is developed for this purpose. An interaction integral for linear piezoelectric materials utilizing the domain-form is implement to extract the relevant dynamic intensity factors. To illustrate the accuracy and efficiency of the proposed method, numerical results for the normalized dynamic intensity factors are presented and compared with the standard XFEM with 
refined mesh and analytical solution. The effects of the combined mechanical and electrical impacts, polarization direction on the normalized dynamic intensity factors is analyzed and discussed in details. Some important features are concluded as follows:

- The numerical results predicted by the V-XFEM agree well with analytical solution and standard XFEM with refined mesh, as expected.

- It can be concluded that the V-XFEM has a higher accuracy and more and efficiency than standard XFEM.

- For piezoelectric structures, numerical results show that the normalized dynamic intensity factors have a significant dependence on the poling direction and electrical impact loading.

- Because of a unified way for all single and coupled linear phenomena, the proposed V-XFEM has the potential to simulate all the linearly physical phenomena.

\section{Acknowledgements}

This work was supported by the National Natural Science Foundation of China (Grant No. 11802261, 11672099, 52075465), the Hunan Science Foundation for Distinguished Young Scholars (Grant No.2019JJ20015), and the science and technology innovation Program of Hunan Province (Grant No. 2020RC4038). The financial supports are gratefully acknowledged.

\section{CRediT authorship contribution statement}

Shuohui Yin: Writing - original draft, Conceptualization, Methodology. Ning Zhang: Programing (elastic problems), Numerical results. Peng Liu: Programing (piezoelectric problems), Numerical results. Jingang Liu: Check and improved the code, review \& editing. Tiantang Yu: Conceptualization, numerical simulation. Shuitao Gu: Writing - review \& editing, Conceptualization, theoretical analysis. Yu Cong: Writing - review \& editing.

\section{References}

[1] Song J H, Wang H, Belytschko T. A comparative study on finite element methods for dynamic fracture. Comput Mech 2008;42:239-250.

[2] Belytschko T, Black T. Elastic crack growth in finite elements with minimal remeshing. Int J 
Numer Methods Eng 1999;45(5):601-20.

[3] Moës N, Dolbow J, Belytschko T. A finite element method for crack growth without remeshing. Int J Numer Methods Eng 1999;46(1):131-50.

[4] Bui T Q, Zhang C. Extended finite element simulation of stationary dynamic cracks in piezoelectric solids under impact loading. Comput Mater Sci 2012;62:243-257.

[5] Liu P, Yu T, Bui Q T, Zhang C. Transient dynamic crack analysis in non-homogeneous functionally graded piezoelectric materials by the X-FEM. Comput Mater Sci 2013;69:542-558.

[6] Liu P, Yu T, Bui T Q, Zhang C, Xu Y, Lim C W. Transient thermal shock fracture analysis of functionally graded piezoelectric materials by the extended finite element method. Int J Solids Struct 2014;51(11-12):2167-82.

[7] Fries T P, Belytschko T. The extended/generalized finite element method: An overview of the method and its applications. Int J Numer Methods Eng 2010;84:253-304.

[8] Li H, Li J, Yuan H. A review of the extended finite element method on macrocrack and microcrack growth simulations. Theor Appl Fract Mech 2018;39:189-207.

[9] Guidault P A, Allix O, Champaney L, Navarro J P. A two-scale approach with homogenization for the computation of cracked structures. Comput Struct 2007;85(17-18):1360-71.

[10] Guidault P A, Allix O, Champaney L, Cornuault C. A multiscale extended finite element method for crack propagation. Comput Methods Appl Mech Eng 2008;197(5):381-99.

[11] Loehnert S, Belytschko T. A multiscale projection method for macro/microcrack simulations. Int J Num Meth Eng 2007;71:1466-82.

[12] Zhou X P, Yang H Q. Multiscale numerical modeling of propagation and coalescence of multiple cracks in rock masses. Int J Rock Mech Min Sci 2012;55:15-27.

[13] Holl M, Rogge T, Loehnert S, Wriggers P, Rolfes R. 3D Multiscale crack propagation using the XFEM applied to a gas turbine blade. Comput Mech 2014;53(1):173-88.

[14] Holl M, Loehnert S, Wriggers P. An adaptive multiscale method for crack propagation and crack coalescence. Int J Numer Methods Eng 2013;93(1):23-51.

[15] Murotani K, Yagawa G, Choi J B. Adaptive finite elements using hierarchical mesh and its application to crack propagation analysis. Comput Meth Appl Mech Eng 2013;253:1-14.

[16] Hettich T, Hund A, Ramm E. Modeling of failure in composites by X-FEM and level sets within a multiscale framework. Comput Meth Appl Mech Eng 2008;197:414-24. 
[17] Ben Dhia H, Jamond O. On the use of XFEM within the Arlequin framework for the simulation of crack propagation. Comput Meth Appl Mech Eng 2010;199:1403-14.

[18] Rannou J, Gravouil A, Baïetto-Dubourg M C. A local multigrid X-FEM strategy for 3-D crack propagation. Int J Numer Methods Eng 2009;77(4):581-600.

[19] Gibert G, Prabel B, Gravouil A, Jacquemoud C. A 3D automatic mesh refinement X-FEM approach for fatigue crack propagation. Finite Elem Anal Des 2019;157:21-37.

[20] Fries T P, Byfut A, Alizada A, Cheng K W, Schröder A. Hanging nodes and XFEM. Int J Numer Methods Eng 2011;86(4-5):404-30.

[21] Budarapu P R, Gracie R, Bordas S P A, Rabczuk T. An adaptive multiscale method for quasi-static crack growth. Comput Mech 2014;53(6):1129-1148.

[22] Patil R U, Mishra B K, Singh I V. A new multiscale XFEM for the elastic properties evaluation of heterogeneous materials. Int J Mech Sci 2017;122:277-87.

[23] Teng Z H, Sun F, Wu S C, Zhang Z B, Chen T, Liao D M. An adaptively refined XFEM with virtual node polygonal elements for dynamic crack problems. Comput Mech 2018;62(5):1087-1106.

[24] Kumar S, Singh I V, Mishra B K, Sharma K, Khan I A. A homogenized multigrid XFEM to predict the crack growth behavior of ductile material in the presence of microstructural defects. Eng Fract Mech 2019;205:577-602.

[25] Lim J H, Sohn D, Im S, Variable-node element families for mesh connection and adaptive mesh computation. Struct Eng Mech 2012;43(3):349-370.

[26] Wang Z, Yu T, Bui T Q, Trinh A N, Nguyen T, Nguyen D D, Doan H D. Numerical modeling of 3-D inclusions and voids by a novel adaptive XFEM. Adv Eng Softw 2016;102:105-22.

[27] Wang Z, Yu T, Bui T Q, Tanaka S, Zhang C, Hirose S, Curiel-Sosa J L. 3-D Local mesh refinement XFEM with variable-node hexahedron elements for extraction of stress intensity factors of straight and curved planar cracks. Comput Methods Appl Mech Eng 2017;313:375-405.

[28] Yu T, Bui T Q. Numerical simulation of 2-d weak and strong discontinuities by a novel approach based on XFEM with local mesh refinement. Comput Struct 2018;196:112-33.

[29] Ding J, Yu T, Yang Y, Bui T Q. An efficient variable-node XFEM for modeling multiple crack growth: A Matlab object-oriented implementation. Adv Eng Softw 2020;140:102750.

[30] Ding J, Yu T, Bui T Q. Modeling strong/weak discontinuities by local mesh refinement variable-node XFEM with object-oriented implementation. Theor Appl Fract Mech 
2020;106:102434.

[31] Han Z, Yu T, Phan H, Bui T Q. Extended stochastic finite element method enhanced by local mesh refinement for random voids analysis. Comput Struct 2020;239:106326.

[32] Ma C, Yu T, Lich L V, Nguyen T, Bui T Q. Detection of multiple complicated flaw clusters by dynamic variable-node XFEM with a three-step detection algorithm. Eur $\mathrm{J}$ Mech A/Solids 2020;82:103980.

[33] Li S H, Mataga P. Dynamic crack propagation in piezoelectric materials. J Mech Phys Solids 1996;44(11):1799-1830.

[34] Enderlein M, Ricoeur A, Kuna M. Finite element techniques for dynamic crack analysis in piezoelectrics, Int J Fract 2005;134:191-208.

[35] Garcia-Sanchez F, Zhang Ch, Sladek J, Sladek V. 2D transient dynamic crack analysis in piezoelectric solids by BEM. Comput Mater Sci 2007;39:179-186.

[36] Garcia-Sanchez F, Zhang Ch, Saez A. 2-D transient dynamic analysis of cracked piezoelectric solids by a time-domain BEM. Comput Methods Appl Mech Engrg 2008;197:3108-3121.

[37] Wunsche M, Garcia-Sanchez F, Saez A, Zhang Ch. A 2D time-domain collocation-Galerkin BEM for dynamic crack analysis in piezoelectric solids. Eng Anal Bound Elem. 2010;34:377-387.

[38] Li C, Song C, Man H, Ooi E T, Gao W. 2D dynamic analysis of cracks and interface cracks in piezoelectric composites using the SBFEM. Int J Solids Struct. 2014;51:2096-2108.

[39] Yu T T, Bui Q T, Liu P, Zhang Ch, Hirose S. Interfacial dynamic impermeable cracks analysis in dissimilar piezoelectric materials under coupled electromechanical loading with the extended finite element method. Int J Solids Struct. 2015;67-68:205-218.

[40] Bechet E, Scherzer M, Kuna M. Application of the X-FEM to the fracture of piezoelectric materials. Int J Numer Methods Eng 2009;77:1535-1565.

[41] Benveniste Y, Milton G W. New exact results for the effective electric, elastic, piezoelectric and other properties of composite ellipsoid assemblages. J. Mech. Phys. Solids 2003;51:1773-1813 [42] Gu S T, He Q C. Interfacial discontinuity relations for coupled multifield phenomena and their application to the modeling of thin interphases as imperfect interfaces. J. Mech. Phys. Solids 2011;59:1413-1426.

[43] Alshits V I, Darinskii A N, Lothe J. On the existence of surface waves in half-infinite anisotropic media with piezoelectric and piezomagnetic properties. Wave Motion 1992;16:265-283. 
[44] Sharma K, Bui Q T, Zhang Ch, Bhargava R R. Analysis of a subinterface crack in piezoelectric bimaterials with the extended finite element method. Eng Fract Mech 2013;104:114-139.

[45] Enderlein M, Ricoeur A, Kuna M. Finite element techniques for dynamic crack analysis in piezoelectrics. Int J Fract 2005;134(3-4):191-208.

[46] Daux C, Moës N, Dolbow J, Sukumar N, Belytschko T. Arbitrary branched and intersecting cracks with the extended finite element method. Int J Numer Methods Eng 2000;48(12):1741 - 60.

[47] Freund L B. Dynamic Fracture Mechanics. Cambridge University Press, London, 1990.

[48] Xu X L, Rajapakse R K N D. A theoretical study of branched cracks in piezoelectrics, Acta. Mater 1999;48:1865-1882.

[49] Lei J, Zhang C. A simplified evaluation of the mechanical energy release rate of kinked cracks in piezoelectric materials using the boundary element method, Eng Fract Mech 2018;188:36-57.

[50] Mishra R, Burela R G. Thermo-electro-mechanical fatigue crack growth simulation in piezoelectric solids using XFEM approach. Theor Appl Fract Mech 2019;104:102388. 